\title{
Fluctuation-dissipation relations in the non-equilibrium critical dynamics of Ising models
}

\author{
Peter Mayer ${ }^{1}$, Ludovic Berthier ${ }^{2,3}$, Juan P. Garrahan ${ }^{2}$ and Peter Sollich ${ }^{1}$ \\ ${ }^{1}$ Department of Mathematics, King's College, Strand, London, WC2R 2LS, UK \\ ${ }^{2}$ Theoretical Physics, University of Oxford, 1 Keble Road, Oxford, OX1 3NP, UK \\ ${ }^{3}$ Laboratoire des Verres, Université Montpellier II, 34095 Montpellier, France
}

(Dated: October 29, 2018)

\begin{abstract}
We investigate the relation between two-time, multi-spin, correlation and response functions in the non-equilibrium critical dynamics of Ising models in $d=1$ and $d=2$ spatial dimensions. In these non-equilibrium situations, the fluctuation-dissipation theorem (FDT) is not satisfied. We find FDT 'violations' qualitatively similar to those reported in various glassy materials, but quantitatively dependent on the chosen observable, in contrast to the results obtained in infinite-range glass models. Nevertheless, all FDT violations can be understood by considering separately the contributions from large wavevectors, which are at quasi-equilibrium and obey FDT, and from small wavevectors where a generalized FDT holds with a non-trivial fluctuation-dissipation ratio $X^{\infty}$. In $d=1$, we get $X^{\infty}=\frac{1}{2}$ for spin observables, which measure the orientation of domains, while $X^{\infty}=0$ for observables that are sensitive to the domain-wall motion. Numerical simulations in $d=2$ reveal a unique $X^{\infty} \simeq 0.34$ for all observables. Measurement protocols for $X^{\infty}$ are discussed in detail. Our results suggest that the definition of an effective temperature $T_{\text {eff }}=T / X^{\infty}$ for large length scales is generically possible in non-equilibrium critical dynamics.

PACS numbers: 05.70.Ln, 75.40.Gb, 75.40.Mg
\end{abstract}

Since the analytical solution of the non-equilibrium dynamics of the spherical $p$-spin model in its low temperature phase [1], many studies have focused on the properties of two-time non-equilibrium correlation and response functions, and the relationship between them [2, [3]. In this paper, we report on analytical and numerical investigations of several two-time, multi-spin, correlation and response functions in the non-equilibrium critical dynamics of Ising models in $d=1$ and $d=2$ spatial dimensions.

Our work is motivated by the following observations. Multi-point dynamical functions are standard objects in equilibrium statistical mechanics which reveal microscopic information related to experimentally observable quantities. In non-equilibrated systems, however, the equilibrium relation between response and correlation, i.e. the fluctuation-dissipation theorem (FDT), is not satisfied. This evident observation became important when it was realized that in the $p$-spin model 1] and more generally in infinite-range glass models, a generalized FDT can be formulated 2, 3, 4, 5]. This amounts to the introduction of a fluctuation-dissipation ratio $X$ or, alternatively, of an effective temperature $T_{\text {eff }}=T / X$ for the slow, non-equilibrated modes of the system [ $[6]$. The properties of $X$ and $T_{\text {eff }}$ have attracted much interest since they suggest that a generalized statistical mechanics can be devised to deal with a broad class of non-equilibrium phenomena.

The generalized FDT is exact for infinite-range glass models only. It is, however, tempting to apply the same concepts in other contexts such as glassy systems with finite interaction range, as observed experimentally or simulated numerically. A further step is made when those ideas are transferred to other physical situations such as domain growth processes [7, 8] in non-disordered systems or the rheology of soft glassy materials $[9,[10]$. Although one does not expect the results for infinite-range glass models to apply exactly in all these non-equilibrium situations, it is worthwhile to understand analogies and differences, and thus to push these ideas as far as possible. This is the philosophy of our paper where non-equilibrium dynamics at criticality is analyzed along the lines described above. Our results suggest that the concept of a generalized FDT is indeed useful at criticality, and we describe in detail the form it takes as compared to the results obtained in infinite-range glass models.

The manuscript is organized as follows. The first section below reviews the results obtained for correlation and response functions in ferromagnets and delineates the scope of the paper. In Sec. III the $1 d$ Ising model is studied analytically at $T_{c}=0$. In Sec. III numerical results for the $2 d$ Ising model at $T_{c}$ are presented. A summary and a physical discussion of the results can be found in Sec. IV] 


\section{FDT AND FERROMAGNETS}

\section{A. Correlation and response functions}

Pure ferromagnets are generally not described as glassy materials, which are loosely defined as systems with large relaxation times. However, if a ferromagnet initially prepared at high temperature is suddenly quenched to its low temperature ferromagnetic phase, its equilibration time diverges with system size [11]. This is true also when the quench is performed precisely to the critical point, $T=T_{c}$. In both cases the system remains, in the thermodynamic limit, forever in a non-equilibrated, nonstationary state: it exhibits aging. Therefore, one can study physical situations in pure ferromagnets that are reminiscent of aging phenomena observed e.g. in spin glasses, polymers or colloids. One is then led to ask if the tools used in the glass literature are also useful to describe this type of non-equilibrium situation.

These tools include, in particular, two-time correlation and response functions. Consider two physical observables $A(t)$ and $B(t)$. Their connected cross-correlation is defined by

$$
C\left(t, t_{\mathrm{w}}\right)=\left\langle A(t) B\left(t_{\mathrm{w}}\right)\right\rangle-\langle A(t)\rangle\left\langle B\left(t_{\mathrm{w}}\right)\right\rangle,
$$

while the conjugate response function is given by

$$
R\left(t, t_{\mathrm{w}}\right)=\left.T \frac{\delta\langle A(t)\rangle}{\delta h_{B}\left(t_{\mathrm{w}}\right)}\right|_{h_{B}=0} .
$$

Here $h_{B}$ is the thermodynamically conjugate field to the observable $B$; for later convenience we scale the response by $T$. Numerically or experimentally, it is often more convenient to measure the integrated response function, or susceptibility,

$$
\chi\left(t, t_{\mathrm{w}}\right)=\int_{t_{\mathrm{w}}}^{t} \mathrm{~d} \tau R(t, \tau),
$$

which gives the response to a small constant field $h_{B}$ switched on at the 'waiting time' $t_{\mathrm{w}}$.

At equilibrium, correlation and response are time-translation invariant and related by the FDT

$$
R\left(t, t_{\mathrm{w}}\right)=\frac{\partial}{\partial t_{\mathrm{w}}} C\left(t, t_{\mathrm{w}}\right)
$$

In that case, $\langle\ldots\rangle$ in Eqs. (12) stands for the usual ensemble average. If one follows instead the dynamics of the system after a sudden quench, the system is out of equilibrium and neither timetranslation invariance nor FDT are satisfied. Then $\langle\ldots\rangle$ is to be read as an average over initial conditions and any stochasticity in the dynamics. In infinite-range glass models, a generalized FDT is satisfied in the aging dynamics. The generalization amounts to the introduction of a fluctuationdissipation ratio $(\mathrm{FDR}), X\left(t, t_{\mathrm{w}}\right)$, through

$$
-\frac{\partial}{\partial t_{\mathrm{w}}} \chi\left(t, t_{\mathrm{w}}\right)=R\left(t, t_{\mathrm{w}}\right)=X\left(t, t_{\mathrm{w}}\right) \frac{\partial}{\partial t_{\mathrm{w}}} C\left(t, t_{\mathrm{w}}\right) .
$$

This definition becomes non-trivial because in the limit of long times $t$ and $t_{\mathrm{w}}$ the FDR reduces to a function of a single variable only, namely the correlation function,

$$
X\left(t, t_{\mathrm{w}}\right) \rightarrow X\left(C\left(t, t_{\mathrm{w}}\right)\right)
$$

where by a an abuse of notation we retain the same symbol for the FDR and its long-time limit. As in equilibrium, response and correlation are then no longer independent quantities, although their relationship is now more complex.

It is now standard to study this generalized FDT via the parametric representation, or 'FD plot', of the susceptibility $\chi$ as a function of the correlation $C$ [4]. At equilibrium, one has $\chi\left(t, t_{\mathrm{w}}\right)=$ $C(t, t)-C\left(t, t_{\mathrm{w}}\right)$. Hence, a plot of $\chi\left(t, t_{\mathrm{w}}\right)$ against $C(t, t)-C\left(t, t_{\mathrm{w}}\right)$ gives a simple straight line of slope 1: this is the equilibrium FD plot. Out of equilibrium, Eq. (6) implies

$$
\chi\left(t, t_{\mathrm{w}}\right)=\int_{C\left(t, t_{\mathrm{w}}\right)}^{C(t, t)} \mathrm{d} x X(x) .
$$


Therefore, when Eq. (6) holds, the FDR can be obtained directly from the slope of the FD plot, which is $X(C)$. Otherwise, from Eq. (5) a plot of $\chi\left(t, t_{\mathrm{w}}\right)$ against $C(t, t)-C\left(t, t_{\mathrm{w}}\right)$, with $t$ fixed and $t_{\mathrm{w}}$ the curve parameter, will still have slope $X\left(t, t_{\mathrm{w}}\right)$. Since the amplitude of correlation and response functions can diverge or converge to zero for $t \rightarrow \infty$ (see below) it can be useful to use normalized quantities, plotting $\tilde{\chi}\left(t, t_{\mathrm{w}}\right) \equiv \chi\left(t, t_{\mathrm{w}}\right) / C(t, t)$ versus $1-\tilde{C}\left(t, t_{\mathrm{w}}\right)$ where $\tilde{C}\left(t, t_{\mathrm{w}}\right)=C\left(t, t_{\mathrm{w}}\right) / C(t, t)$. Since the normalization factors are independent of $t_{\mathrm{w}}$, the slope of the FD plot is then still given by $X$. The normalization issue is less important when presenting numerical data, which are by construction obtained in a restricted time window where the amplitudes of the dynamical quantities typically change only slowly.

Appealingly, the FDR can also be interpreted as defining an effective temperature, $T_{\text {eff }}\left(t, t_{\mathrm{w}}\right) \equiv$ $T / X\left(t, t_{\mathrm{w}}\right)$, replacing the equilibrium temperature $T$ by an equivalent quantity out of equilibrium. Moreover, it is a general result that for the case $A=B$, where one considers the autocorrelation of $A$ and the associated response, $T_{\text {eff }}$ is the temperature measured by a thermometer coupled to the observable $A$ at the appropriate timescale [6]. As a direct corollary, this effective temperature then satisfies the zeroth law of thermodynamics. Clearly, however, the introduction of an effective temperature is of thermodynamic interest only if $T_{\text {eff }}$ is actually independent of the observables $A$ and $B$ under consideration. This is indeed true for infinite-range glass models [6], implying that although the system is out of equilibrium it can still be described in thermodynamic terms, at the moderate cost of introducing one extra parameter, namely the effective temperature 12]. Beyond infinite-range glass models, the observable-dependence of the effective temperature remains largely an open question but has been discussed in detail in the context of trap models [13] and in a realistic numerical model of a supercooled liquid [14].

\section{B. Ferromagnets at low temperature}

For ferromagnets, two-time dynamical functions have been studied both for a quench to the low temperature phase and to criticality, with most work to date focused on the first situation.

In the low temperature phase, the evolution of the system consists in the growth of ordered domains, with a typical domain size $\ell(t)$. Two-time quantities that have been thoroughly studied are the spin autocorrelation function, $C_{\mathrm{s}}\left(t, t_{\mathrm{w}}\right)=\left\langle s_{i}(t) s_{i}\left(t_{\mathrm{w}}\right)\right\rangle$, where $s_{i}(t)$ is the value of the spin at site $i$ at time $t$, and the conjugate response function, $R_{\mathrm{s}}\left(t, t_{\mathrm{w}}\right)=-\left(\partial / \partial t_{\mathrm{w}}\right) \chi_{\mathrm{s}}\left(t, t_{\mathrm{w}}\right)=\delta\left\langle s_{i}(t)\right\rangle / \delta h_{i}\left(t_{\mathrm{w}}\right)$, where $h_{i}$ is the magnetic field at site $i$. In this case, the connected and disconnected correlations coincide since $\left\langle s_{i}(t)\right\rangle=0$ at all stages of the coarsening process. From the analytical solution of solvable models [15, 16, 17, 18, 19] and the simulation of more realistic situations [7, 8, 20, 21, 22], the behavior of these two quantities is now well known. For small time differences, $\Delta t=t-t_{\mathrm{w}} \ll t_{\mathrm{w}}$, time-translation invariant behavior is observed, $C_{\mathrm{s}}\left(t, t_{\mathrm{w}}\right) \approx C_{\mathrm{s}}(\Delta t), \chi_{\mathrm{s}}\left(t, t_{\mathrm{w}}\right) \approx \chi_{\mathrm{s}}(\Delta t)$, and the FDT $\chi_{\mathrm{s}}\left(t, t_{\mathrm{w}}\right) \approx C_{\mathrm{s}}(t, t)-C_{\mathrm{s}}\left(t, t_{\mathrm{w}}\right)$ is obeyed. This first regime is due to thermal fluctuations in the bulk of the domains, which are essentially equilibrium fluctuations. For larger time separations, $\Delta t \gg t_{\mathrm{w}}$, the fluctuations of the interfaces dominate the dynamical behavior. The reasonable hypothesis that coarsening is a self-similar process, in the sense that for large times all dynamical functions depend on time only through the typical domain size $\ell$, implies the scaling form $C_{\mathrm{s}}\left(t, t_{\mathrm{w}}\right) \approx C_{\mathrm{s}}\left(\ell(t) / \ell\left(t_{\mathrm{w}}\right)\right)$ for the correlation function. The contribution of the interfaces to the response function can be estimated $[8]$ as a sum over all wavevectors, $\chi_{\mathrm{s}}\left(t, t_{\mathrm{w}}\right) \approx \int_{1 / \ell\left(t_{\mathrm{w}}\right)}^{1 / a} \mathrm{~d}^{d} \mathbf{k} k^{-2} \ell^{-1}(t)$. This expression results from the fact that the response at time $t_{\mathrm{w}}$ is dominated by large wavevectors, $k \ell\left(t_{\mathrm{w}}\right) \gg 1$, each wavevector $\mathbf{k}$ giving a contribution of the order of $k^{-2}$. The factor $\ell^{-1}(t)$ represents the density of domain walls, and $a$ is a UV cutoff given by the lattice spacing. This reasoning implies for the long-time contribution to the susceptibility the scaling form $\chi_{\mathrm{s}}\left(t, t_{\mathrm{w}}\right) \approx f\left(\ell\left(t_{\mathrm{w}}\right)\right) \chi_{\mathrm{s}}\left(\ell(t) / \ell\left(t_{\mathrm{w}}\right)\right)$, where the function $f(x)$ depends on the dimensionality $d$ of space and is given by $f(x)=1 / x$ for $d>2, f(x)=(\ln x) / x$ for $d=2$ and $f(x)=$ const for $d=1$. This scaling function has recently been revisited in Refs. [22, 23], with particular attention to the case $d=2$ [24, 25].

From the above arguments, and for $d>1$, the parametric plot of $\chi_{\mathrm{s}}\left(t, t_{\mathrm{w}}\right)$ versus $C_{\mathrm{s}}\left(t, t_{\mathrm{w}}\right)-C_{\mathrm{s}}\left(t, t_{\mathrm{w}}\right)$ consists of an initial equilibrium part followed by an essentially horizontal section. In the latter the correlation function decays due to interface motion, while the response function hardly changes because any contribution from the interfaces is suppressed by the $f\left(\ell\left(t_{\mathrm{w}}\right)\right)$ prefactor. If a limiting FDR, $X_{\mathrm{s}}^{\infty}$, is defined through

$$
X_{\mathrm{s}}^{\infty}=\lim _{t_{\mathrm{w}} \rightarrow \infty} \lim _{t \rightarrow \infty} X_{\mathrm{s}}\left(t, t_{\mathrm{w}}\right)
$$


then it follows that $X_{\mathrm{s}}^{\infty}=0$ for $d>1$ in coarsening processes. For $d=1$, on the other hand, both $\chi_{\mathrm{s}}$ and $C_{\mathrm{s}}$ are scaling functions of $\ell(t) / \ell\left(t_{\mathrm{w}}\right)$ and the parametric plot assumes no simple shape, implying that $X_{\mathrm{s}}^{\infty}$ could be any finite number. This is confirmed by the analytical solution of the dynamics of the Ising chain at $T=0$ which shows that $X_{\mathrm{s}}^{\infty}=\frac{1}{2}\left[26\right.$, 27]. The factor $\frac{1}{2}$ was first derived in Ref. [28].

\section{Ferromagnets at the critical point}

The non-trivial value of $X_{\mathrm{s}}^{\infty}$ for the Ising chain was interpreted using the fact that in $d=1$, the ordering temperature $T=0$ coincides with the critical point, $T_{c}=0$ [26]. It was then suggested that a non-trivial $X_{\mathrm{s}}^{\infty}$ could be a generic feature of critical points [29]. This is physically reasonable, since the whole argument for $X_{\mathrm{s}}^{\infty}=0$ in coarsening processes relies on the separation between bulk and interfaces; this is no longer valid at the critical point where the bulk has the well-known self-similar structure of ferromagnets at criticality.

From analytical and numerical studies, the behavior of two-time single-spin dynamical quantities is again well understood, as briefly reviewed in Ref. [30]. Physically, the non-equilibrium dynamics following a quench to the critical point consists in the growth of the dynamical correlation length, $\xi(t) \approx t^{1 / z}$, where $z$ is the dynamical critical exponent [31]. Critical fluctuations of large wavevectors, $k \xi(t) \gg 1$, are almost equilibrated, while those with small wavevectors, $k \xi(t) \ll 1$, still retain their non-equilibrium initial condition. This separation leads to the scaling forms $C_{\mathrm{s}}\left(t, t_{\mathrm{w}}\right) \approx \Delta t^{-2 \beta / \nu z} C_{\mathrm{s}}\left(\xi(t) / \xi\left(t_{\mathrm{w}}\right)\right)$ and $\chi_{\mathrm{s}}\left(t, t_{\mathrm{w}}\right) \approx \Delta t^{-2 \beta / \nu z} \chi_{\mathrm{s}}\left(\xi(t) / \xi\left(t_{\mathrm{w}}\right)\right)$, where $\beta$ and $\nu$ are the standard critical exponents. This can be interpreted as follows. For short time differences $\Delta t \ll t_{\mathrm{w}}$, equilibrated fluctuations with large $k$ dominate and dynamical functions assume their standard equilibrium power-law decay. The dynamics at large time separation $\Delta t \gg t_{\mathrm{w}}$, on the other hand, is dominated by the growth of the dynamic correlation length and leads to the $\xi(t) / \xi\left(t_{\mathrm{w}}\right)$ scaling. This in turn implies that, beyond the initial equilibrium part, the FD plot will again assume a non-trivial shape, as in the Ising chain. The striking similarity of these results with the aging dynamics of finite dimensional spin glasses was noted in Refs. [29, 32].

The reasoning above confirms that at criticality $X_{\mathrm{s}}^{\infty}$ can take any finite value, in contrast with the $X_{\mathrm{s}}^{\infty}=0$ obtained in the low temperature phase. It was further argued that $X_{\mathrm{s}}^{\infty}$ should be a new universal quantity at criticality 29]. As such, it can be computed using standard renormalization group procedures, and this program has recently been started for various models 33, 34, 35]. The value of $X_{\mathrm{s}}^{\infty}$ is known exactly for the Ising chain [26, 27, 28], where $X_{\mathrm{s}}^{\infty}=\frac{1}{2}$, for the spherical ferromagnetic model [29], where $X_{\mathrm{s}}^{\infty}=\frac{1}{2}$ for $d \geq 4$ and $X_{\mathrm{s}}^{\infty}=\frac{1}{3}$ for $d=3$, and for the Gaussian model [15] where $X_{\mathrm{s}}^{\infty}=\frac{1}{2}$. An estimate is known for model A at second order in $4-d$ [33], to first order in $\sqrt{4-d}$ for the diluted Ising model [34], and to first order in $4-d$ in model C [35].

\section{Motivations for this work}

This short review of known results in the non-equilibrium dynamics of pure ferromagnets shows that much research has been done on the subject. So, why another paper?

First of all, the relevance of the notion of an effective temperature at criticality can be questioned because the FD plots for the spin dynamic functions do not assume a simple linear shape with a well-defined slope, as happens in the low temperature phase. This is related to the fact that at low temperatures the decay of correlation functions occurs on two well-separated time scales. Each has its own associated effective temperature, a fact reminiscent of the physics of structural glasses. At criticality, on the other hand, one has a continuum of time scales associated with different wavevectors, $t(k) \sim k^{-z}$. Moreover, for finite $k$ the equilibration time is finite, meaning that the number of modes that are still out of equilibrium decreases as time increases. This suggests that an effective temperature could be relevant only when considering the $k \rightarrow 0$ limit, a point which our analysis will clarify.

Second, we mentioned the important issue of the observable-dependence of a generalized FDT. This issue remains completely open since the studies cited above focused exclusively on a single FD relation, for the spin autocorrelation and associated response. In order to get a more complete theoretical understanding it is crucial to understand if other observables give the same results, and if not, how they are related. 
A third motivation for the study of higher order correlation functions comes from the observation that the dynamics of coarsening models is dominated by the motion of topological defects. For Ising models, these are domain walls, the local density of which is given by the 'defect' observable $s_{i}(t) s_{j}(t)$, where $(i, j)$ are nearest neighbors. Defect dynamical functions have recently been studied in the context of kinetically constrained Ising models [36], and the FD relations that arose showed interesting and unexpected features.

For an Ising model, there are at least four 'natural' FD relations, involving respectively the spin autocorrelation, $C_{\mathrm{s}}\left(t, t_{\mathrm{w}}\right)$, the magnetization density $m(t)$ correlation, $C_{\mathrm{m}}\left(t, t_{\mathrm{w}}\right)=\left\langle m(t) m\left(t_{\mathrm{w}}\right)\right\rangle$, the defect autocorrelation, $C_{\mathrm{d}}\left(t, t_{\mathrm{w}}\right)=\left\langle s_{i}(t) s_{j}(t) s_{i}\left(t_{\mathrm{w}}\right) s_{j}\left(t_{\mathrm{w}}\right)\right\rangle-\left\langle s_{i}(t) s_{j}(t)\right\rangle\left\langle s_{i}\left(t_{\mathrm{w}}\right) s_{j}\left(t_{\mathrm{w}}\right)\right\rangle$ with $(i, j)$ nearest neighbors, and the energy density $e(t)$ correlation, $C_{\mathrm{e}}\left(t, t_{\mathrm{w}}\right)=\left\langle e(t) e\left(t_{\mathrm{w}}\right)\right\rangle-\langle e(t)\rangle\left\langle e\left(t_{\mathrm{w}}\right)\right\rangle$. Note again that connected and disconnected correlation functions coincide for the magnetization; this is not the case for $C_{\mathrm{d}}\left(t, t_{\mathrm{w}}\right)$ and $C_{\mathrm{e}}\left(t, t_{\mathrm{w}}\right)$. In the $1 d$ case, we will also investigate two-time functions which smoothly interpolate between incoherent, local functions (spin or defect) and coherent, global ones (magnetization, energy), and discuss the case of correlation functions of higher order. In the $2 d$ case, we will stick to the four quantities listed above.

\section{THE $1 d$ ISING MODEL}

In this section we study the non-equilibrium dynamics in the Glauber-Ising chain with Hamiltonian

$$
\mathcal{H}=-\sum_{i} s_{i} s_{i+1}
$$

where the $s_{i}(i=1, \ldots N)$ are $N$ Ising spins subject to periodic boundary conditions. Glauber dynamics consists in each spin $s_{i}$ flipping with rate $\frac{1}{2}\left[1-\frac{1}{2} \gamma s_{i}\left(s_{i-1}+s_{i+1}\right)\right]$, where $\gamma=\tanh (2 / T)$. We focus on the evolution of arbitrary two-time spin and defect correlation and response functions in the thermodynamic limit $N \rightarrow \infty$, after a quench from equilibrium at $T=\infty$ to $T \rightarrow 0$. As explained above, although a variety of aspects of the associated coarsening dynamics have already been studied [1, 37], results on the non-equilibrium FDT violation so far are restricted to the spin autocorrelation and response functions [26, 27]. In Sec. [A we introduce the more general class of spin and defect observables we investigate. We briefly present the main result of our method to derive multi-spin two-time correlation and response functions, as developed in Ref. [38], and summarize the approach used to extract from this the quantities of interest here. Our results for spin dynamical functions are then given in Sec. IIB - a preliminary account of which has appeared in the conference proceedings [39] - and for defect functions in Sec. IIC In Sec. IID we discuss the physical aspects of our results for the $1 d$ Ising model.

\section{A. General strategy for the calculations}

We consider the following spin and defect observables $O_{\mathrm{s}}$ and $O_{\mathrm{d}}$

$$
O_{\mathrm{s}}=\sum_{i} \epsilon_{i} s_{i} \quad \text { and } \quad O_{\mathrm{d}}=\sum_{i} \epsilon_{i} s_{i} s_{i+1}
$$

In both cases the $\epsilon_{i}$ are quenched random variables with zero mean $\left[\epsilon_{i}\right]=0$ and translation invariant covariances $q_{i-j}=\left[\epsilon_{i} \epsilon_{j}\right]$; here [ $\left.\cdot\right]$ denotes the average over the distribution of $\epsilon$. Without loss of generality we set $q_{0}=1$. We define the corresponding connected two-time correlation functions

$$
C\left(t, t_{\mathrm{w}}\right)=\frac{1}{N}\left[\left\langle O_{\mathrm{s}}(t) O_{\mathrm{s}}\left(t_{\mathrm{w}}\right)\right\rangle\right] \quad \text { and } \quad C\left(t, t_{\mathrm{w}}\right)=\frac{1}{N}\left[\left\langle O_{\mathrm{d}}(t) O_{\mathrm{d}}\left(t_{\mathrm{w}}\right)\right\rangle-\left\langle O_{\mathrm{d}}(t)\right\rangle\left\langle O_{\mathrm{d}}\left(t_{\mathrm{w}}\right)\right\rangle\right],
$$

for spins and defects, respectively, and the responses

$$
R\left(t, t_{\mathrm{w}}\right)=\left.\frac{T}{N}\left[\frac{\delta\left\langle O_{\mathrm{s}}(t)\right\rangle}{\delta h_{\mathrm{s}}\left(t_{\mathrm{w}}\right)}\right]\right|_{h_{\mathrm{s}}=0} \quad \text { and } \quad R\left(t, t_{\mathrm{w}}\right)=\left.\frac{T}{N}\left[\frac{\delta\left\langle O_{\mathrm{d}}(t)\right\rangle}{\delta h_{\mathrm{d}}\left(t_{\mathrm{w}}\right)}\right]\right|_{h_{\mathrm{d}}=0},
$$

where $h_{\mathrm{s}}$ and $h_{\mathrm{d}}$ are thermodynamically conjugate to $O_{\mathrm{s}}$ and $O_{\mathrm{d}}$, respectively. All functions are scaled by $N$ to get quantities of order unity. It is easy to show that, in the thermodynamic limit $N \rightarrow \infty$, 
Eqs. (11), (12) become 38]

$$
C\left(t, t_{\mathrm{w}}\right)=\sum_{n} q_{n} C_{n}\left(t, t_{\mathrm{w}}\right) \quad \text { and } \quad R\left(t, t_{\mathrm{w}}\right)=\sum_{n} q_{n} R_{n}\left(t, t_{\mathrm{w}}\right) .
$$

Here we have used translational invariance (which holds for our quench from an equilibrium state) to define the distance-dependent correlation functions

$$
\begin{aligned}
& C_{j-i}\left(t, t_{\mathrm{w}}\right)=\left\langle s_{i}(t) s_{j}\left(t_{\mathrm{w}}\right)\right\rangle \quad(\text { spins }), \\
& C_{j-i}\left(t, t_{\mathrm{w}}\right)=\left\langle s_{i}(t) s_{i+1}(t) s_{j}\left(t_{\mathrm{w}}\right) s_{j+1}\left(t_{\mathrm{w}}\right)\right\rangle-\left\langle s_{i}(t) s_{i+1}(t)\right\rangle\left\langle s_{j}\left(t_{\mathrm{w}}\right) s_{j+1}\left(t_{\mathrm{w}}\right)\right\rangle \quad(\text { defects }),
\end{aligned}
$$

and associated responses

$$
R_{j-i}\left(t, t_{\mathrm{w}}\right)=\left.T \frac{\delta\left\langle s_{i}(t)\right\rangle}{\delta h_{j}\left(t_{\mathrm{w}}\right)}\right|_{h_{j}=0}(\text { spins }) \quad \text { and } \quad R_{j-i}\left(t, t_{\mathrm{w}}\right)=\left.T \frac{\delta\left\langle s_{i}(t) s_{i+1}(t)\right\rangle}{\delta h_{j, j+1}\left(t_{\mathrm{w}}\right)}\right|_{h_{j, j+1}=0} \text { (defects). }
$$

As usual, $h_{j}$ and $h_{j, j+1}$ are conjugate to $s_{j}$ and $s_{j} s_{j+1}$, respectively. Translation invariance also shows that in the thermodynamic limit $N \rightarrow \infty$, the expressions (11), (12) are self-averaging, i.e. independent of the particular realization of the disorder variables $\epsilon_{i}$.

Analysis of the non-equilibrium FDR for the observables $O_{\mathrm{s}}, O_{\mathrm{d}}$ thus requires knowledge of all spin and defect correlation and response functions (14), (15). We have tackled this problem in [38] where we give closed, exact solutions for generic two-time multi-spin correlation and response functions in the Glauber-Ising chain after a quench from an arbitrary equilibrium state at temperature $T_{\mathrm{i}}>0$ to any $T \geq 0$. The approach is based on the closed hierarchy of differential equations for the spincorrelations $\left\langle s_{i_{1}}(t) s_{i_{2}}(t) \cdots s_{i_{k}}(t)\right\rangle$, which we solved for arbitrary initial correlations $\left\langle s_{i_{1}}(0) \cdots s_{i_{k}}(0)\right\rangle$. The key result reads [38]

$$
\begin{gathered}
\left\langle s_{i_{1}}(t) \cdots s_{i_{k}}(t)\right\rangle=\sum_{l=0}^{\left\lfloor\frac{k}{2}\right\rfloor} \sum_{\pi \in \mathcal{P}(l, k)}(-1)^{\pi} \prod_{\lambda=1}^{l} \mathrm{H}_{i_{\pi(2 \lambda)}-i_{\pi(2 \lambda-1)}}(2 t) \Phi_{\left(i_{\pi(2 l+1)}, \ldots i_{\pi(k)}\right)}^{(k-2 l)}(t) \\
\Phi_{i}^{(k)}(t)=\sum_{j_{1}<\ldots j_{k}}\left(\sum_{\pi \in \mathcal{S}(k)}(-1)^{\pi} \prod_{\lambda=1}^{k} \mathrm{e}^{-t} \mathrm{I}_{i_{\lambda}-j_{\pi(\lambda)}}(\gamma t)\right)\left\langle s_{j_{1}}(0) \cdots s_{j_{k}}(0)\right\rangle
\end{gathered}
$$

Here $\pi$ denotes permutations and $(-1)^{\pi}$ their $\operatorname{sign} ; \mathcal{S}(k)$ is the set of all permutations of $\{1,2, \ldots k\}$ while $\mathcal{P}(l, k)$ is the set of permutations corresponding to choosing $l$ ordered pairs from the numbers $1,2, \ldots k$ and keeping the remaining $k-2 l$ numbers in ascending order. Explicit expressions for the functions $\mathrm{I}_{n}(x)$ and $\mathrm{H}_{n}(x)$ are given in 38] (for $N \rightarrow \infty$ the $\mathrm{I}_{n}(x)$ are modified Bessel functions, see Appendix A). We also show in 38 that the evolution of two-time, multi-spin correlation and response functions is governed by an identical hierarchy of differential equations, so that these quantities can be obtained from (16), (17) if we substitute the corresponding equal-time initial conditions in (17). The latter are just equal-time correlations — or can be expressed in terms of these for the response functions - which we know already. For a quench from an equilibrium state this leads to explicit results for the two-time multi-spin functions. As simple examples we state in [38] the spin and defect functions (14), (15) for the quench from $T_{\mathrm{i}}=\infty$ to $T \rightarrow 0$ considered here. For spins one finds

$$
\begin{aligned}
& C_{n}\left(t, t_{\mathrm{w}}\right)=\mathrm{e}^{-\left(t+t_{\mathrm{w}}\right)}\left\{\mathrm{I}_{n}\left(t+t_{\mathrm{w}}\right)+\int_{0}^{2 t_{\mathrm{w}}} \mathrm{d} \tau \mathrm{I}_{n}\left(t+t_{\mathrm{w}}-\tau\right)\left[\mathrm{I}_{0}+\mathrm{I}_{1}\right](\tau)\right\} \\
& \chi_{n}\left(t, t_{\mathrm{w}}\right)=\frac{1}{2} \mathrm{e}^{-t} \int_{t_{\mathrm{w}}}^{t} \mathrm{~d} \tau \mathrm{e}^{-\tau} \mathrm{I}_{n}(t-\tau)\left[\mathrm{I}_{0}+2 \mathrm{I}_{1}+\mathrm{I}_{2}\right](2 \tau),
\end{aligned}
$$

and for defects

$$
\begin{aligned}
C_{n}\left(t, t_{\mathrm{w}}\right) & =\frac{1}{2} \mathrm{e}^{-\left(t+t_{\mathrm{w}}\right)}\left[\mathrm{I}_{n-1}-\mathrm{I}_{n+1}\right]\left(t+t_{\mathrm{w}}\right) \int_{t-t_{\mathrm{w}}}^{t+t_{\mathrm{w}}} \mathrm{d} \tau \mathrm{e}^{-\tau}\left[\mathrm{I}_{n-1}-\mathrm{I}_{n+1}\right](\tau) \\
& +\mathrm{e}^{-2 t}\left\{\mathrm{I}_{n}\left(t-t_{\mathrm{w}}\right)\left[\mathrm{I}_{n-1}+2 \mathrm{I}_{n}+\mathrm{I}_{n+1}\right]\left(t+t_{\mathrm{w}}\right)-\mathrm{e}^{-2 t_{\mathrm{w}}}\left[\left(\mathrm{I}_{n-1}+\mathrm{I}_{n}\right)\left(\mathrm{I}_{n}+\mathrm{I}_{n+1}\right)\right]\left(t+t_{\mathrm{w}}\right)\right\}, \\
\chi_{n}\left(t, t_{\mathrm{w}}\right) & =\mathrm{e}^{-2 t}\left\{2 \delta_{n, 0}\left[\mathrm{I}_{0}+\mathrm{I}_{1}\right](2 t)-\mathrm{I}_{n}\left(t-t_{\mathrm{w}}\right)\left[\mathrm{I}_{n-1}+2 \mathrm{I}_{n}+\mathrm{I}_{n+1}\right]\left(t+t_{\mathrm{w}}\right)\right\} .
\end{aligned}
$$


Here and below the short-hand $[\ldots](x)$ is used to indicate that all functions enclosed in the square brackets have the same argument $x ; \delta_{n, 0}$ is the standard Kronecker delta. The expressions (19), (21) for the susceptibilities are more convenient than those for the responses $R_{n}\left(t, t_{\mathrm{w}}\right)=-\left(\partial / \partial t_{\mathrm{w}}\right) \chi_{n}\left(t, t_{\mathrm{w}}\right)$ and so we mostly base the following discussion on them. We note that while equations (18), (19) have already been given in various forms, e.g. [26], we are not aware of any equivalent in the literature of (20), (21).

Eqs. (18)- (21) will form the basis for our analysis of the FDR in the $1 d$ Ising chain in Secs. IIB and IIC For now we return to the observables $O_{\mathrm{s}}, O_{\mathrm{d}}$ and in particular the choice of the field covariances $q_{n}$. According to (13) we obtain spin and defect autocorrelation and response functions by choosing uncorrelated random fields $\epsilon_{i}$, i.e. $q_{n}=\delta_{n, 0}$. We abbreviate the notation in this case to that used in the introduction and write $C_{\mathrm{s}}\left(t, t_{\mathrm{w}}\right)$ for spin and $C_{\mathrm{d}}\left(t, t_{\mathrm{w}}\right)$ for defect autocorrelations and similarly $\chi_{\mathrm{s}}\left(t, t_{\mathrm{w}}\right), \chi_{\mathrm{d}}\left(t, t_{\mathrm{w}}\right)$ for susceptibilities. Uniform covariances $q_{n}=1$, on the other hand, yield full summations over all cross-correlation and response functions in (13). So $O_{\mathrm{s}}$ and $O_{\mathrm{d}}$ produce just the magnetization and energy, respectively; we thus use the obvious short-hands $C_{\mathrm{m}}\left(t, t_{\mathrm{w}}\right), \chi_{\mathrm{m}}\left(t, t_{\mathrm{w}}\right)$, and $C_{\mathrm{e}}\left(t, t_{\mathrm{w}}\right), \chi_{\mathrm{e}}\left(t, t_{\mathrm{w}}\right)$ for this case. It will turn out that the local $\left(q_{n}=\delta_{n, 0}\right)$ and global $\left(q_{n}=\right.$ 1) FDT relations for spin and defect observables are very different. Therefore we also investigate intermediate choices of $q_{n}$ that interpolate between these two extremes. Two classes of covariances can be distinguished. We may interpolate between $q_{n}=\delta_{n, 0}$ and $q_{n}=1$ by a family of covariances that satisfies $\sum_{n}\left|q_{n}\right|<\infty$ for any non-uniform choice of $q_{n}$; we call the corresponding fields $\epsilon_{i}$ shortrange correlated. Alternatively, we can interpolate such that $\sum_{n}\left|q_{n}\right|=\infty$ as long as the fields are not completely uncorrelated; we refer to such fields as infinite-range correlated. In either case the analysis of the FDR for the correlation and response functions of the associated observable requires us to evaluate the infinite sums in (13). This can be done conveniently in terms of the Fourier transforms $q(k)=\mathcal{F}\left\{q_{n}\right\}, C\left(k ; t, t_{\mathrm{w}}\right)=\mathcal{F}\left\{C_{n}\left(t, t_{\mathrm{w}}\right)\right\}$ and $\chi\left(k ; t, t_{\mathrm{w}}\right)=\mathcal{F}\left\{\chi_{n}\left(t, t_{\mathrm{w}}\right)\right\}$ where

$$
\mathcal{F}\left\{f_{n}\right\}=\sum_{n} f_{n} \mathrm{e}^{-i n k} \quad \text { and } \quad \mathcal{F}^{-1}\{f(k)\}=\int_{-\pi}^{\pi} \frac{\mathrm{d} k}{2 \pi} f(k) \mathrm{e}^{i n k} .
$$

In Appendix [B] we state the Fourier transforms of (18)-(21), in terms of which (13) becomes

$$
C\left(t, t_{\mathrm{w}}\right)=\int_{-\pi}^{\pi} \frac{\mathrm{d} k}{2 \pi} q(k) C\left(k ; t, t_{\mathrm{w}}\right) \quad \text { and } \quad \chi\left(t, t_{\mathrm{w}}\right)=\int_{-\pi}^{\pi} \frac{\mathrm{d} k}{2 \pi} q(k) \chi\left(k ; t, t_{\mathrm{w}}\right) .
$$

An explicit example of a family of short-range correlated fields, parameterized by $a>0$, is given by the Lorentzian covariances

$$
q_{\mathrm{L}, n}=\frac{a^{2}}{a^{2}+n^{2}} \quad \Leftrightarrow \quad q_{\mathrm{L}}(k)=\frac{a \pi}{\sinh a \pi} \cosh a(\pi-|k|) .
$$

The transform (24) can be found in any table of Fourier transforms. Eq. (24) indeed defines shortrange correlated fields: since $q_{\mathrm{L}, n}>0$ the criterion becomes $N_{\mathrm{c}} \equiv \sum_{n} q_{\mathrm{L}, n}<\infty$ which is satisfied since $N_{\mathrm{c}}=q_{\mathrm{L}}(0)=a \pi \operatorname{coth} a \pi$. By varying $a$ we can also smoothly tune our observables between local $\left(q_{\mathrm{L}, n} \rightarrow \delta_{n, 0}\right.$ as $\left.a \rightarrow 0\right)$ and global $\left(q_{\mathrm{L}, n} \rightarrow 1\right.$ for $\left.a \rightarrow \infty\right)$ ones. We denote the corresponding correlations and susceptibilities by $C_{\mathrm{L}}\left(t, t_{\mathrm{w}}\right)$ and $\chi_{\mathrm{L}}\left(t, t_{\mathrm{w}}\right)$. An example of covariances that yield infinite-range correlated fields is

$$
q_{\mathrm{P}, n}=(-1)^{n} \frac{\Gamma^{2}\left(\frac{1+\alpha}{2}\right)}{\Gamma\left(\frac{1+\alpha}{2}-n\right) \Gamma\left(\frac{1+\alpha}{2}+n\right)} \quad \Leftrightarrow \quad q_{\mathrm{P}}(k)=\frac{\Gamma^{2}\left(\frac{1+\alpha}{2}\right)}{2^{1-\alpha} \Gamma(\alpha)}\left|\sin \frac{k}{2}\right|^{\alpha-1},
$$

where $0<\alpha<1$ and $\Gamma(x)$ is the Gamma function [40]. It is clear from (25) that $q_{\mathrm{P}, n}$ is even in $n$ and $q_{\mathrm{P}, 0}=1$. We show in Appendix C that for $\alpha \rightarrow 1$ we get $q_{\mathrm{P}, n}=\delta_{n, 0}$ while $\alpha \rightarrow 0$ gives $q_{\mathrm{P}, n}=1$. We also prove there that $q_{\mathrm{P}, n}$ decreases monotonically as $|n|$ increases, decaying asymptotically as a power-law $q_{\mathrm{P}, n} \sim|n|^{-\alpha}$, and that indeed $\mathcal{F}^{-1}\left\{q_{\mathrm{P}}(k)\right\}=q_{\mathrm{P}, n}$. The reverse transform $\mathcal{F}\left\{q_{\mathrm{P}, n}\right\}$ does not converge in the usual sense, but this is not necessary for equivalence of (13) and (23). So (25) again allows us to interpolate smoothly between local and global observables, but in such a way that $\sum_{n} q_{\mathrm{P}, n}=\infty$ for any $\alpha \in[0,1[$. The correlations and susceptibilities for the observables defined by fields $\epsilon_{i}$ with the power-law covariances (25) are denoted by $C_{\mathrm{P}}\left(t, t_{\mathrm{w}}\right)$ and $\chi_{\mathrm{P}}\left(t, t_{\mathrm{w}}\right)$ below. 


\section{B. Spin observables}

\section{Random field: Incoherent functions}

The FDT violation for the spin autocorrelation and response function has already been studied in detail in [26, 27]. In particular it was shown that the FD plot approaches a non-trivial limit curve in the aging regime, with $X_{\mathrm{s}}^{\infty}=\frac{1}{2}$. We can easily recover the existing results for $C_{\mathrm{s}}\left(t, t_{\mathrm{w}}\right), \chi_{\mathrm{s}}\left(t, t_{\mathrm{w}}\right)$ from our exact solutions (18), (19) by setting $n=0$. It is useful to focus on the aging limit. Formally this is an asymptotic expansion in the limit $t, t_{\mathrm{w}} \rightarrow \infty$ with $\epsilon \leq t_{\mathrm{w}} / t \leq 1-\delta$ fixed and $\epsilon, \delta>0$, to ensure that $t, t_{\mathrm{w}}$ and $\Delta t$ all diverge and are of the same order. In this limit the asymptotic expansion (A3) for modified Bessel functions yields immediately

$$
\begin{aligned}
& C_{\mathrm{s}}\left(t, t_{\mathrm{w}}\right) \sim \frac{2}{\pi} \arcsin \sqrt{\frac{2 t_{\mathrm{w}}}{t+t_{\mathrm{w}}}}, \\
& \chi_{\mathrm{s}}\left(t, t_{\mathrm{w}}\right) \sim \frac{\sqrt{2}}{\pi} \arccos \sqrt{\frac{t_{\mathrm{w}}}{t}} .
\end{aligned}
$$

Here and below, the ' $\sim$ ' sign denotes results which are asymptotically exact in the aging limit. The limit FD-plot corresponding to (26), (27) is contained in Fig. 2 below and the associated FDR is a function of the time ratio $t_{\mathrm{w}} / t$ only,

$$
X_{\mathrm{s}}\left(t, t_{\mathrm{w}}\right) \sim \frac{t+t_{\mathrm{w}}}{2 t} .
$$

It shows a continuous crossover from $X_{\mathrm{s}}\left(t, t_{\mathrm{w}}\right)=1$ for $\Delta t \ll t_{\mathrm{w}}$ to $X_{\mathrm{s}}\left(t, t_{\mathrm{w}}\right)=X_{\mathrm{s}}^{\infty}=\frac{1}{2}$ for $\Delta t \gg t_{\mathrm{w}}$. We note that the aging expansion of the spin correlations and susceptibilities (18), (19) is dominated by the leading term of the asymptotic series (A3) for the modified Bessel functions, which is independent of the order $n$. Therefore (26), (27) in fact apply to all finite-distance spin cross-correlations and susceptibilities $C_{n}\left(t, t_{\mathrm{w}}\right), \chi_{n}\left(t, t_{\mathrm{w}}\right)$. Consequently, the latter produce the same limiting FD plot and FDR (28) as for $n=0$.

\section{Uniform field: Coherent functions}

As described, the uniform field effectively allows us to study the FDR for the magnetization. The corresponding correlation and susceptibility are most conveniently obtained from the Fourier transforms (B1), (B2) by setting $k=0$; the time integrals appearing in $C\left(k ; t, t_{\mathrm{w}}\right), \chi\left(k ; t, t_{\mathrm{w}}\right)$ can then be solved. One finds

$$
\begin{aligned}
& C_{\mathrm{m}}\left(t, t_{\mathrm{w}}\right)=\mathrm{e}^{-2 t_{\mathrm{w}}}\left\{\mathrm{I}_{0}\left(2 t_{\mathrm{w}}\right)+4 t_{\mathrm{w}}\left[\mathrm{I}_{0}+\mathrm{I}_{1}\right]\left(2 t_{\mathrm{w}}\right)\right\} \\
& R_{\mathrm{m}}\left(t, t_{\mathrm{w}}\right)=\frac{1}{2} \mathrm{e}^{-2 t_{\mathrm{w}}}\left[\mathrm{I}_{0}+2 \mathrm{I}_{1}+\mathrm{I}_{2}\right]\left(2 t_{\mathrm{w}}\right) .
\end{aligned}
$$

We have given the response $R=-\partial \chi / \partial t_{\mathrm{w}}$ here rather than the susceptibility because it has a simpler form. Note that both the correlation and response function (29), (30) are independent of $t$. This can be understood from the fact that for $T=0$ the magnetization $m=(1 / N) \sum_{i} s_{i}$ performs a random walk with step size $\pm 2 / N$ and a time-dependent rate $\frac{1}{2} N c$, where $c$ is the concentration of domain walls. The latter can be obtained explicitly from (18) by setting $t=t_{\mathrm{w}}$ and $n=1$, since $C_{1}\left(t_{\mathrm{w}}, t_{\mathrm{w}}\right)=\left\langle s_{i}\left(t_{\mathrm{w}}\right) s_{i+1}\left(t_{\mathrm{w}}\right)\right\rangle=1-2 c\left(t_{\mathrm{w}}\right)$. This gives [41]

$$
c\left(t_{\mathrm{w}}\right)=\frac{1}{2} \mathrm{e}^{-2 t_{\mathrm{w}}}\left[\mathrm{I}_{0}+\mathrm{I}_{1}\right]\left(2 t_{\mathrm{w}}\right)
$$

Now, since the random walk of $m$ is unbiased, $C_{\mathrm{m}}\left(t, t_{\mathrm{w}}\right)=N\left\langle m(t) m\left(t_{\mathrm{w}}\right)\right\rangle=C_{\mathrm{m}}\left(t_{\mathrm{w}}, t_{\mathrm{w}}\right)$ follows immediately. Also, $C_{\mathrm{m}}\left(t_{\mathrm{w}}, t_{\mathrm{w}}\right)$ will grow with rate $2 N D\left(t_{\mathrm{w}}\right)$, where $D=(2 / N)^{2} \frac{1}{2} N c=2 c / N$ is the diffusion constant. One should thus have $\partial C_{\mathrm{m}}\left(t_{\mathrm{w}}, t_{\mathrm{w}}\right) / \partial t_{\mathrm{w}}=4 c\left(t_{\mathrm{w}}\right)$, and from (29), (31) one verifies that this is indeed the case. Similar arguments apply to the response $R_{\mathrm{m}}\left(t, t_{\mathrm{w}}\right)$. Brief application of a field at $t_{\mathrm{w}}$ biases the domain wall motion and hence the random walk of $m$; thereafter the random walk is again unbiased and so the response must be $t$-independent. The momentary bias in the 
random walk rates contains the domain wall concentration $c\left(t_{\mathrm{w}}\right)$ as an overall factor, and consistent with this expectation one gets $R_{\mathrm{m}}\left(t, t_{\mathrm{w}}\right) \sim 2 c\left(t_{\mathrm{w}}\right)$ asymptotically.

Since both $C_{\mathrm{m}}\left(t, t_{\mathrm{w}}\right)$ and $R_{\mathrm{m}}\left(t, t_{\mathrm{w}}\right)$ are functions of $t_{\mathrm{w}}$ only, this also applies to the FDR

$$
X_{\mathrm{m}}\left(t_{\mathrm{w}}\right)=\frac{\left[\mathrm{I}_{0}+2 \mathrm{I}_{1}+\mathrm{I}_{2}\right]\left(2 t_{\mathrm{w}}\right)}{4\left[\mathrm{I}_{0}+\mathrm{I}_{1}\right]\left(2 t_{\mathrm{w}}\right)},
$$

which crosses over from the initial value $X_{\mathrm{m}}(0)=\frac{1}{4}$ to $\frac{1}{2}$ on an $\mathcal{O}(1)$ time scale. So, apart from a transient after the quench, we measure $X_{\mathrm{m}}\left(t_{\mathrm{w}}\right)=\frac{1}{2}$ for all $t \geq t_{\mathrm{w}} \gg 1$; in particular the limiting value $X_{\mathrm{m}}\left(t_{\mathrm{w}}\right) \sim X_{\mathrm{m}}^{\infty}=X_{\mathrm{s}}^{\infty}=1 / 2$ in the aging regime is identical to that for the incoherent spin observables. Note that there is no quasi-equilibrium regime with $X_{\mathrm{m}}=1$ for $\Delta t \ll t_{\mathrm{w}}$. The corresponding FD plot converges to a straight line of slope $\frac{1}{2}$ (see Fig. 2 below).

\section{Short-range correlated field}

Next we investigate the effect of short-range correlations in the random fields $\epsilon_{i}$ on the FDR. The correlations and susceptibilities of the corresponding observables may be obtained either from a realspace summation (13) or an integration in the Fourier representation (23). Using the latter, we note first that the short-range criterion $\sum_{n}\left|q_{n}\right|<\infty$ for the covariances implies that $q(k)=\mathcal{F}\left\{q_{n}\right\}=$ $\sum_{n} q_{n} \mathrm{e}^{-i n k}$ is a continuous function. The Fourier transforms $C\left(k ; t, t_{\mathrm{w}}\right), \chi\left(k ; t, t_{\mathrm{w}}\right)$, on the other hand, satisfy

$$
\frac{C\left(k ; t, t_{\mathrm{w}}\right)}{C_{\mathrm{s}}\left(t, t_{\mathrm{w}}\right)} \rightarrow 2 \pi \tilde{\delta}(k) \quad \text { and } \quad \frac{\chi\left(k ; t, t_{\mathrm{w}}\right)}{\chi_{\mathrm{s}}\left(t, t_{\mathrm{w}}\right)} \rightarrow 2 \pi \tilde{\delta}(k)
$$

in the aging limit, where $\tilde{\delta}(\cdot)$ is a $2 \pi$-periodic version of the ordinary Dirac delta. The normalizations of the right-hand sides of (33) are clear since $C_{\mathrm{s}}\left(t, t_{\mathrm{w}}\right)$, for instance, is given by the Fourier integral (22) over $C\left(k ; t, t_{\mathrm{w}}\right)$ for $n=0$. And since $C_{\mathrm{s}}\left(t, t_{\mathrm{w}}\right)$ and $\chi_{\mathrm{s}}\left(t, t_{\mathrm{w}}\right)$ are $\mathcal{O}(1)$ functions of $t_{\mathrm{w}} / t$ in the aging limit, while $C\left(k ; t, t_{\mathrm{w}}\right), \chi\left(k ; t, t_{\mathrm{w}}\right)$ vanish in the same limit for any $k$ that is not a multiple of $2 \pi$, Eq. (33) follows. This in turn implies that for any short-range correlated field

$$
C\left(t, t_{\mathrm{w}}\right) \sim N_{\mathrm{c}} C_{\mathrm{s}}\left(t, t_{\mathrm{w}}\right) \quad \text { and } \quad \chi\left(t, t_{\mathrm{w}}\right) \sim N_{\mathrm{c}} \chi_{\mathrm{s}}\left(t, t_{\mathrm{w}}\right)
$$

provided that $N_{\mathrm{c}}=q(0)=\sum_{n} q_{n}$, which estimates the number of lattice sizes over which field correlations extend, is nonzero. So in the aging limit the correlations and susceptibilities are ultimately just proportional to (26), (27) and hence yield the same FDR and FD plot as the local spin functions. This statement can equivalently be made in real space, based on convergence of the series $q_{n}$ and the fact that all finite-distance cross-correlation and response functions behave asymptotically as (26), (27).

At finite times, however, we find a crossover between two dynamical regimes. A scaling analysis shows that the peaks in $C\left(k ; t, t_{\mathrm{w}}\right)$ and $\chi\left(k ; t, t_{\mathrm{w}}\right)$ at $k=0$ have widths $t^{-1 / 2}$ and $\Delta t^{-1 / 2}$, respectively. Correspondingly, we have growing length scales in real space. These are $\ell_{C} \approx t^{1 / 2}$ for correlations, corresponding to the typical domain size, but $\ell_{\chi} \approx \Delta t^{1 / 2}$ for the response which reflects the fact that perturbations spread diffusively. When $\ell_{C}, \ell_{\chi} \gg N_{\mathrm{c}}$, one has an effectively local observable and we are in the asymptotic regime (34). If, however, $\ell_{C}, \ell_{\chi} \ll N_{\mathrm{c}}$, the fields $\epsilon_{i}$ are correlated over distances much longer than the dynamical length scales, giving an effectively uniform field. One thus expects to get an FD plot similar to that obtained for the magnetization. The illustration of the crossover in Fig. 11 obtained by numerical integration of (23), shows that this is indeed the case.

\section{Infinite-range correlated field}

For infinite-range correlated fields one cannot use simple scaling arguments since the correlations and susceptibilities contain contributions from all length scales. For the power-law covariances (25) introduced above, this is reflected in the singularity of $q_{\mathrm{P}}(k)$ at $k=0$. Therefore we have to analyze the full expressions for $C_{\mathrm{P}}\left(t, t_{\mathrm{w}}\right)$ and $\chi_{\mathrm{P}}\left(t, t_{\mathrm{w}}\right)$ that follow from (23) after substitution of $q_{\mathrm{P}}(k)$, $C\left(k ; t, t_{\mathrm{w}}\right)$ and $\chi\left(k ; t, t_{\mathrm{w}}\right)$. Fortunately, for the particular choice of $q_{\mathrm{P}}(k)$ the results may be expressed 

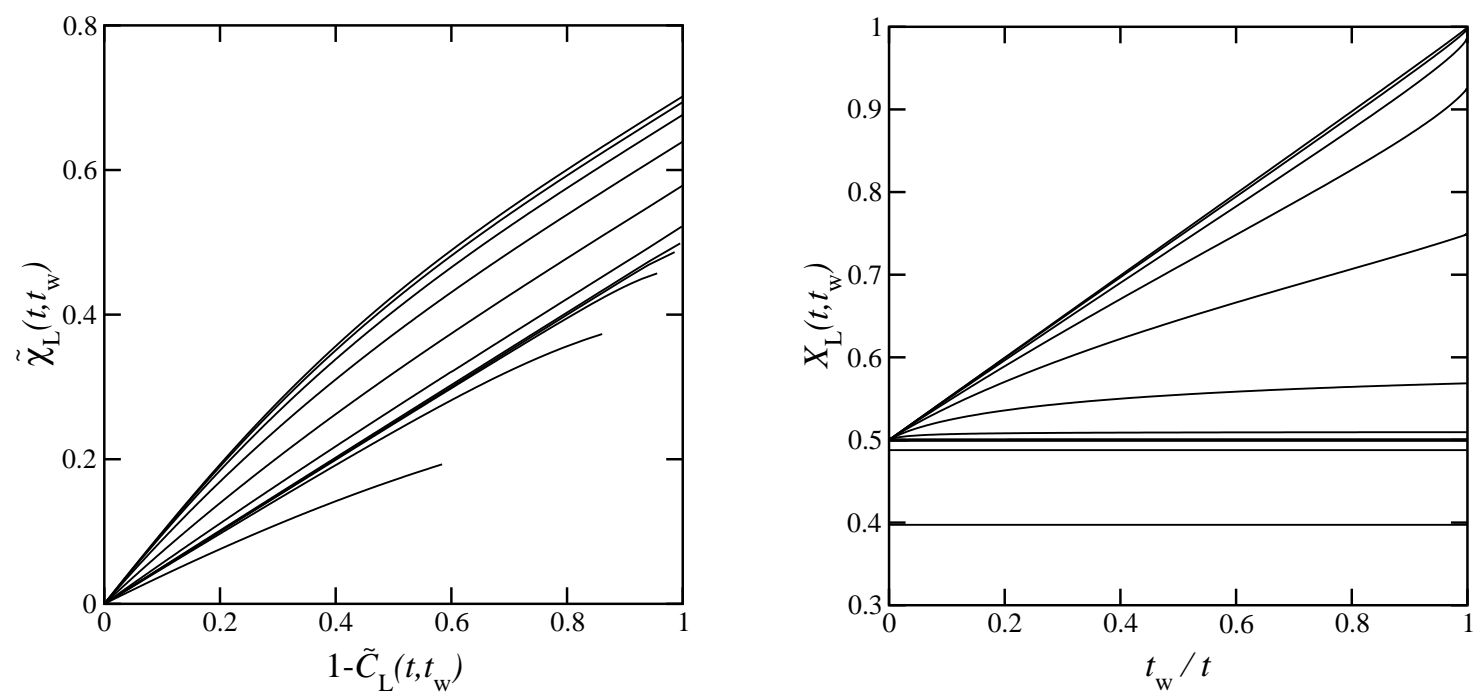

FIG. 1: Normalized FD plot (left) and the corresponding FDR versus $t_{\mathrm{w}} / t$ (right) for a random field with Lorentzian covariances (24) with $a=10^{3}$, correlated over $N_{c}=a \pi \operatorname{coth} a \pi \approx a \pi$ sites. In the FD plot, $t$ is fixed for each curve and varies over the range $10^{0}, 10^{1}, \ldots 10^{10}$ (bottom to top). The lines first converge towards the straight line with slope $\frac{1}{2}$ corresponding to a coherent observable (the magnetization) but eventually, for $t \geq 10^{5}$, cross over to the limit plot for uncorrelated fields. This behaviour is also reflected in the evolution of $X_{\mathrm{L}}\left(t, t_{\mathrm{w}}\right)$. There, however, we have the freedom to fix either $t$ or $t_{\mathrm{w}}$. The plot shows the case of fixed $t_{\mathrm{w}}$, which is more convenient for comparison with simulations, for $t_{\mathrm{w}}=10^{0}, \ldots 10^{10}$ (bottom to top).

in terms of single integrals of the form

$$
\begin{aligned}
& C_{\mathrm{P}}\left(t, t_{\mathrm{w}}\right)=\mathrm{e}^{-2\left(t+t_{\mathrm{w}}\right)}\left\{{ }_{1} \mathrm{~F}_{1}\left(\frac{1}{2}, \frac{1+\alpha}{2} ; 2\left(t+t_{\mathrm{w}}\right)\right)+\int_{0}^{2 t_{\mathrm{w}}} \mathrm{d} \tau \mathrm{e}^{\tau}{ }_{1} \mathrm{~F}_{1}\left(\frac{1}{2}, \frac{1+\alpha}{2} ; 2\left(t+t_{\mathrm{w}}-\tau\right)\right)\left[\mathrm{I}_{0}+\mathrm{I}_{1}\right](\tau)\right\}, \\
& \chi_{\mathrm{P}}\left(t, t_{\mathrm{w}}\right)=\frac{1}{2} \mathrm{e}^{-2 t} \int_{t_{\mathrm{w}}}^{t} \mathrm{~d} \tau_{1} \mathrm{~F}_{1}\left(\frac{1}{2}, \frac{1+\alpha}{2} ; 2(t-\tau)\right)\left[\mathrm{I}_{0}+2 \mathrm{I}_{1}+\mathrm{I}_{2}\right](2 \tau),
\end{aligned}
$$

where ${ }_{1} \mathrm{~F}_{1}(\alpha, \gamma ; z)$ is the confluent hypergeometric function [40]. Equations. (35), (36) are exact and can be used to study the FD plots and the FDR numerically. However, in the aging limit asymptotic expansions may be substituted for the non-elementary functions and significant simplifications are possible. One finds

$$
\begin{aligned}
& C_{\mathrm{P}}\left(t, t_{\mathrm{w}}\right) \sim \frac{2^{\frac{1-\alpha}{2}}}{\pi} \Gamma\left(\frac{1+\alpha}{2}\right)\left(t+t_{\mathrm{w}}\right)^{\frac{1-\alpha}{2}} \mathrm{~B}\left(\frac{1}{2}, 1-\frac{\alpha}{2} ; \frac{2 t_{\mathrm{w}}}{t+t_{\mathrm{w}}}\right), \\
& \chi_{\mathrm{P}}\left(t, t_{\mathrm{w}}\right) \sim \frac{2^{-\frac{\alpha}{2}}}{\pi} \Gamma\left(\frac{1+\alpha}{2}\right) t^{\frac{1-\alpha}{2}}\left[\mathrm{~B}\left(\frac{1}{2}, 1-\frac{\alpha}{2}\right)-\mathrm{B}\left(\frac{1}{2}, 1-\frac{\alpha}{2} ; \frac{t_{\mathrm{w}}}{t}\right)\right],
\end{aligned}
$$

where $\mathrm{B}(p, q ; x)$ is the incomplete Beta function $\mathrm{B}(p, q ; x)=\int_{0}^{x} \mathrm{~d} u u^{p-1}(1-u)^{q-1}$ and $\mathrm{B}(p, q)=$ $\mathrm{B}(p, q ; 1)$ is the complete one [40]. In the random field limit, $\alpha \rightarrow 1$, we recover the expansions (26), (27) for the incoherent functions since $\mathrm{B}\left(\frac{1}{2}, \frac{1}{2} ; x\right)=2 \arcsin \sqrt{x}$, whereas the uniform field limit $\alpha \rightarrow 0$ can be shown to coincide, using $\mathrm{B}\left(\frac{1}{2}, 1 ; x\right)=2 \sqrt{x}$, with the asymptotic expansions of (29), (30) for the coherent functions. So the power-law covariances (25) indeed allow us to interpolate between the coherent and incoherent observables. For intermediate exponents $0<\alpha<1$ the fluctuations in the observable $O_{\mathrm{s}}$ grow as $t_{\mathrm{w}}^{(1-\alpha) / 2}$ and the two-time correlation (37) has a plateau at a corresponding value for $\Delta t \ll t_{\mathrm{w}}$; for $\Delta t \gg t_{\mathrm{w}}$ it decays as $t_{\mathrm{w}}^{(1-\alpha) / 2}\left(t_{\mathrm{w}} / \Delta t\right)^{\alpha / 2}$. For the susceptibility we deduce from (38) a $\Delta t^{(1-\alpha) / 2}\left(\Delta t / t_{\mathrm{w}}\right)^{1 / 2}$ growth for $\Delta t \ll t_{\mathrm{w}}$ that crosses over to $\Delta t^{(1-\alpha) / 2}$ for $\Delta t \gg t_{\mathrm{w}}$. Fig. 2 shows exact FD limit plots that follow from (37), (38). The associated FDR may be obtained 
from (37), 38) as

$$
X_{\mathrm{P}}\left(t, t_{\mathrm{w}}\right) \sim\left\{\frac{2 t}{t+t_{\mathrm{w}}}+\frac{1-\alpha}{2} \sqrt{\frac{2 t_{\mathrm{w}}}{t+t_{\mathrm{w}}}}\left(\frac{t-t_{\mathrm{w}}}{t+t_{\mathrm{w}}}\right)^{\frac{\alpha}{2}} \mathrm{~B}\left(\frac{1}{2}, 1-\frac{\alpha}{2} ; \frac{2 t_{\mathrm{w}}}{t+t_{\mathrm{w}}}\right)\right\}^{-1} .
$$

In principle one should first differentiate (35), (36) to obtain $R_{\mathrm{P}}\left(t, t_{\mathrm{w}}\right)$ and $\left(\partial / \partial t_{\mathrm{w}}\right) C_{\mathrm{P}}\left(t, t_{\mathrm{w}}\right)$ and then perform the aging expansion, but this turns out to give the same result. Equation (39) is a function of $t_{\mathrm{w}} / t$ only and interpolates between the FDR (28) for the local spin observables $(\alpha \rightarrow 1)$ and the constant $X_{\mathrm{m}}^{\infty}=\frac{1}{2}$ for the magnetization $(\alpha \rightarrow 0)$. Plots of $X_{\mathrm{P}}\left(t, t_{\mathrm{w}}\right)$ for various powers $\alpha$ are also shown in Fig. 22 It is remarkable that the FDR again crosses over from $X_{\mathrm{P}}\left(t, t_{\mathrm{w}}\right)=1$ for $\Delta t \ll t_{\mathrm{w}}$ to $X_{\mathrm{P}}^{\infty}=\frac{1}{2}$ for $\Delta t \gg t_{\mathrm{w}}$, independently of the power-law exponent $\alpha$.
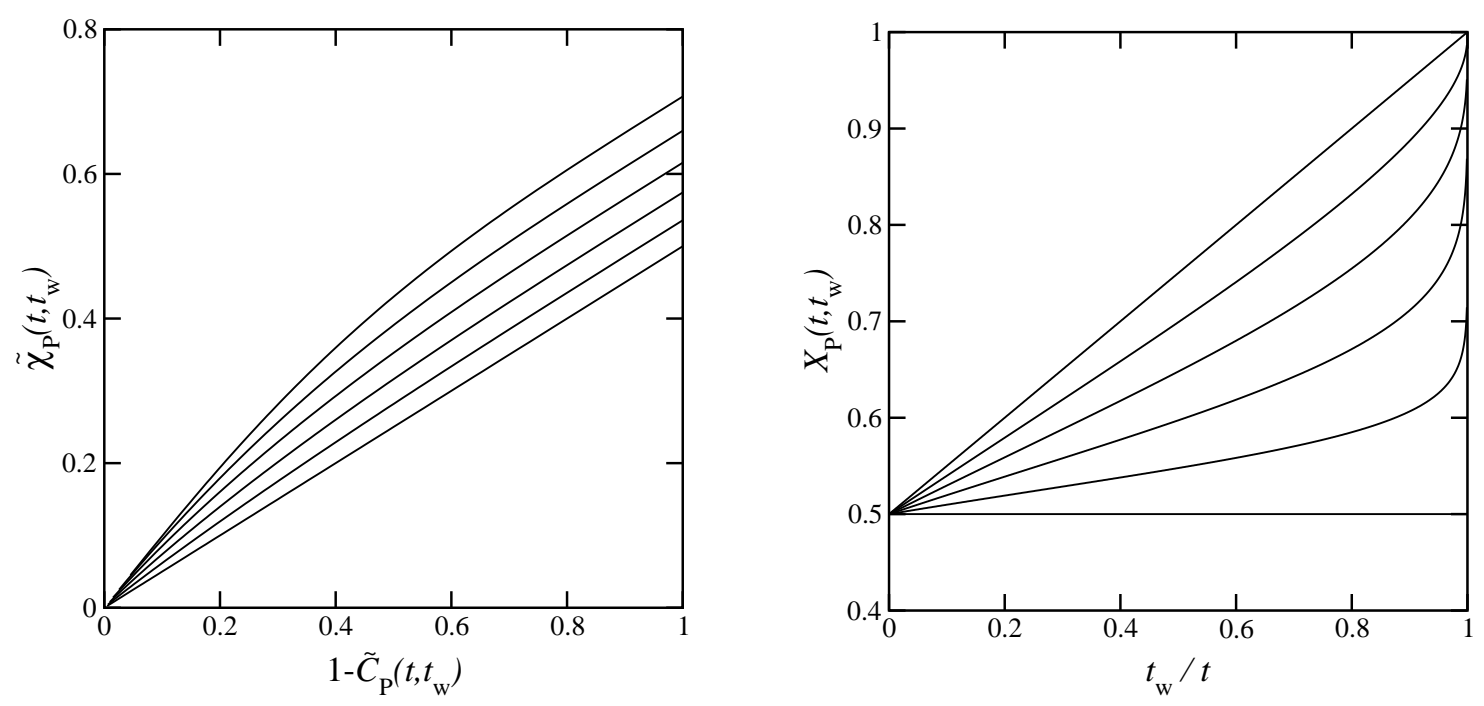

FIG. 2: Normalized FD-plots (left) and the corresponding FDR versus $t_{\mathrm{w}} / t$ (right) in the aging limit $t, t_{\mathrm{w}} \rightarrow$ $\infty$. The bottom curves in both plots are for the magnetization (Sec. IIB 2) and coincide with the uniform field limit $\alpha \rightarrow 0$ of power-law field covariances. The intermediate curves are for power-laws (Sec. IIB 4) with exponents $\alpha=0.2,0.4,0.6,0.8$ (bottom to top). The top curves represent the random field limit $\alpha \rightarrow 1$ for power-laws, and apply also to any short-range correlated field (Sec. IIB3) with $N_{\mathrm{c}} \neq 0$ or, in the extreme case, the incoherent functions of Sec. IIB 1

\section{Harmonically correlated fields and $X^{\infty}$}

The explicit examples given in Sec. IIB 1IIB 4 suggest that $X^{\infty}=\frac{1}{2}$ is a generic feature for the spin-observables $O_{\mathrm{s}}$ defined in (10). To show that this is indeed true, we start from the fact that for a general observable $O_{\mathrm{s}}$ the correlation and susceptibility - and hence $\left(\partial / \partial t_{\mathrm{w}}\right) C\left(t, t_{\mathrm{w}}\right)$ and $R\left(t, t_{\mathrm{w}}\right)$ may be written in the form (23). By introducing a generalized FDT for the Fourier modes

$$
R\left(k ; t, t_{\mathrm{w}}\right)=X\left(k ; t, t_{\mathrm{w}}\right) \frac{\partial}{\partial t_{\mathrm{w}}} C\left(k ; t, t_{\mathrm{w}}\right),
$$

we may express $R\left(k ; t, t_{\mathrm{w}}\right)$ via (40) and thereby obtain the following representation for the FDR $X\left(t, t_{\mathrm{w}}\right)$ associated with a generic spin-observable $O_{\mathrm{s}}$ :

$$
X\left(t, t_{\mathrm{w}}\right)=\frac{\int_{-\pi}^{\pi} \frac{\mathrm{d} k}{2 \pi} X\left(k ; t, t_{\mathrm{w}}\right) q(k) \frac{\partial}{\partial t_{\mathrm{w}}} C\left(k ; t, t_{\mathrm{w}}\right)}{\int_{-\pi}^{\pi} \frac{\mathrm{d} k}{2 \pi} q(k) \frac{\partial}{\partial t_{\mathrm{w}}} C\left(k ; t, t_{\mathrm{w}}\right)} .
$$

This means that $X\left(t, t_{\mathrm{w}}\right)$ may be considered as the average of $X\left(k ; t, t_{\mathrm{w}}\right)$ over the normalized distribution of $q(k)\left(\partial / \partial t_{\mathrm{w}}\right) C\left(k ; t, t_{\mathrm{w}}\right)$ on $k \in[-\pi, \pi]$. The FDR for Fourier modes follows from (40) and 
the expressions (B1), (B2) for $C\left(k ; t, t_{\mathrm{w}}\right), \chi\left(k ; t, t_{\mathrm{w}}\right)$ as

$$
X\left(k ; t_{\mathrm{w}}\right)=\frac{\left[\mathrm{I}_{0}+2 \mathrm{I}_{1}+\mathrm{I}_{2}\right]\left(2 t_{\mathrm{w}}\right)}{4\left[\mathrm{I}_{0}+\mathrm{I}_{1}\right]\left(2 t_{\mathrm{w}}\right)-2(1-\cos k)\left\{\mathrm{e}^{2 t_{\mathrm{w}} \cos k}+\int_{0}^{2 t_{\mathrm{w}}} \mathrm{d} \tau \mathrm{e}^{\left(2 t_{\mathrm{w}}-\tau\right) \cos k}\left[\mathrm{I}_{0}+\mathrm{I}_{1}\right](\tau)\right\}},
$$

and is a function of $t_{\mathrm{w}}$ and $k$ only. For $k=0$, (42) reduces to the FDR for the magnetization $X_{\mathrm{m}}\left(t_{\mathrm{w}}\right)$ (32) and hence $X\left(0 ; t_{\mathrm{w}}\right) \approx \frac{1}{2}$ for $t_{\mathrm{w}} \gg 1$. A scaling analysis of (42) shows that for $|k| \ll \pi$ and $t_{\mathrm{w}} \gg 1$ we get $X\left(k ; t_{\mathrm{w}}\right) \approx X\left(k^{2} t_{\mathrm{w}}\right)$ with $X\left(k^{2} t_{\mathrm{w}}\right) \approx \frac{1}{2}$ for $k^{2} t_{\mathrm{w}} \ll 1$ and $X\left(k^{2} t_{\mathrm{w}}\right) \approx 1$ when $k^{2} t_{\mathrm{w}} \gg 1$. So (42) reflects the successive equilibration of increasing length scales.

Now we can return to the FDR (41) for the observable $O_{\mathrm{s}}$. For the magnetization - being the coherent observable - we have $q(k)=2 \pi \tilde{\delta}(k)$ and (41) reduces to the trivial identity $X_{\mathrm{m}}\left(t_{\mathrm{w}}\right)=$ $X\left(0 ; t_{\mathrm{w}}\right)$. In physical terms, by selecting the coherent observable we only measure the FDR associated with the infinite length scale. For other spin-observables, being characterized by the function $q(k)$, the FDR $X\left(t, t_{\mathrm{w}}\right)$ contains contributions from all length scales. For the long-time limit $X^{\infty}$, however, the situation simplifies because $\left(\partial / \partial t_{\mathrm{w}}\right) C\left(k ; t, t_{\mathrm{w}}\right)$ develops an infinitely sharp peak at $k=0$ as $t \rightarrow \infty$. This can be verified by a scaling analysis of (B1). For sufficiently well-behaved functions $q(k)$, the normalized version of the distribution $q(k)\left(\partial / \partial t_{\mathrm{w}}\right) C\left(k ; t, t_{\mathrm{w}}\right)$ thus becomes a realization of $\tilde{\delta}(k)$ and we get $X\left(t, t_{\mathrm{w}}\right) \rightarrow X\left(0 ; t_{\mathrm{w}}\right)$ as $t \rightarrow \infty$. Taking the limit $t_{\mathrm{w}} \rightarrow \infty$ then shows that $X^{\infty}=\frac{1}{2}$, as claimed. So for a generic spin observable, $X^{\infty}$ again just gives the FDR associated with the infinite length scale. The only exception occurs when this contribution is explicitly suppressed. An example of the latter case would be harmonically correlated fields, $q_{n}=\cos n p$ with $0<p<\pi$ : for such observables $X\left(t, t_{\mathrm{w}}\right)=X\left(p ; t_{\mathrm{w}}\right)$ and hence $X^{\infty}=1$.

\section{Defect observables}

\section{Random field: Incoherent functions}

The defect observable $O_{\mathrm{d}}$ given in (10) with random, uncorrelated fields $\epsilon_{i}$ allows us to study the FDT violation for local defect correlations and susceptibilities. These follow from (20), (21) by setting $n=0$, giving

$$
\begin{aligned}
& C_{\mathrm{d}}\left(t, t_{\mathrm{w}}\right)=2 \mathrm{e}^{-2 t} \mathrm{I}_{0}\left(t-t_{\mathrm{w}}\right)\left[\mathrm{I}_{0}+\mathrm{I}_{1}\right]\left(t+t_{\mathrm{w}}\right)-\mathrm{e}^{-2\left(t+t_{\mathrm{w}}\right)}\left[\mathrm{I}_{0}+\mathrm{I}_{1}\right]^{2}\left(t+t_{\mathrm{w}}\right), \\
& \chi_{\mathrm{d}}\left(t, t_{\mathrm{w}}\right)=2 \mathrm{e}^{-2 t}\left\{\left[\mathrm{I}_{0}+\mathrm{I}_{1}\right](2 t)-\mathrm{I}_{0}\left(t-t_{\mathrm{w}}\right)\left[\mathrm{I}_{0}+\mathrm{I}_{1}\right]\left(t+t_{\mathrm{w}}\right)\right\}
\end{aligned}
$$

These results can be written in a more physically intuitive way in terms of the concentration of domain walls $c(t)$, Eq. (31), and the return probability $p_{\mathrm{r}}(\tau)=\mathrm{e}^{-\tau} \mathrm{I}_{0}(\tau)$ of a continuous-time random walker on a discrete, one-dimensional lattice [42]. Expressing all time dependencies in (43), (44) via $c(t)$ and $p_{\mathrm{r}}(\tau)$ yields the exact identities

$$
\begin{aligned}
& C_{\mathrm{d}}\left(t, t_{\mathrm{w}}\right)=4 c\left(\frac{t+t_{\mathrm{w}}}{2}\right)\left[p_{\mathrm{r}}\left(t-t_{\mathrm{w}}\right)-c\left(\frac{t+t_{\mathrm{w}}}{2}\right)\right], \\
& \chi_{\mathrm{d}}\left(t, t_{\mathrm{w}}\right)=4\left[c(t)-p_{\mathrm{r}}\left(t-t_{\mathrm{w}}\right) c\left(\frac{t+t_{\mathrm{w}}}{2}\right)\right] .
\end{aligned}
$$

The fact that we find random walk-related quantities does not come as a surprise given that there is an exact mapping of zero temperature Glauber dynamics in the Ising chain to a diffusion-limited pair annihilation (DLPA) process [43]. The mapping follows by assigning to each bond $(i, i+1)$ the 'particle' occupation number $b_{i}=\frac{1}{2}\left(1-s_{i} s_{i+1}\right) \in\{0,1\}$ which signals the presence or absence of a domain wall. Glauber dynamics for the spins corresponds to independent random walks for the particles and coalescence of domains of aligned spins yields particle pair-annihilation.

It follows from the definition (14) of the defect autocorrelation that $C_{\mathrm{d}}\left(t, t_{\mathrm{w}}\right)=$ $4\left(\left\langle b_{i}(t) b_{i}\left(t_{\mathrm{w}}\right)\right\rangle-\left\langle b_{i}(t)\right\rangle\left\langle b_{i}\left(t_{\mathrm{w}}\right)\right\rangle\right)$ in fact also describes the particle autocorrelation in the DLPA process. We note that (45) is a non-trivial result. Assuming as in Ref. [36] that the autocorrelation of the fraction $c(t)$ of particles that still exist at time $t$ is given by $p_{\mathrm{r}}\left(t-t_{\mathrm{w}}\right)$ and that these particles are uncorrelated with the fraction $c\left(t_{\mathrm{w}}\right)-c(t)$ of particles that have disappeared via annihilation, one would conclude $\left\langle b_{i}(t) b_{i}\left(t_{\mathrm{w}}\right)\right\rangle=c(t) p_{\mathrm{r}}\left(t-t_{\mathrm{w}}\right)+c(t)\left(c\left(t_{\mathrm{w}}\right)-c(t)\right)$ and hence $C_{\mathrm{d}}\left(t, t_{\mathrm{w}}\right)=4 c(t)\left(p_{\mathrm{r}}\left(t-t_{\mathrm{w}}\right)-c(t)\right)$. This obviously differs from the exact solution (45). As an approximation it holds for $\Delta t \ll t_{\mathrm{w}}$, but breaks down for $\Delta t \gg t_{\mathrm{w}}$ where (45) yields $C_{\mathrm{d}}\left(t, t_{\mathrm{w}}\right) \approx 2 t_{\mathrm{w}} /\left(\pi \Delta t^{2}\right)$ whereas the approximation gives $C_{\mathrm{d}}\left(t, t_{\mathrm{w}}\right) \approx(\sqrt{2}-1) /(\pi \Delta t)$. This shows that two-time correlations in $C_{\mathrm{d}}\left(t, t_{\mathrm{w}}\right)$ build up via a rather 
subtle mechanism, the explanation of which in terms of DLPA would probably require knowledge of the inter-particle (i.e. domain size) distribution. Similarly, it appears that the result (46) cannot be obtained in a straightforward way.
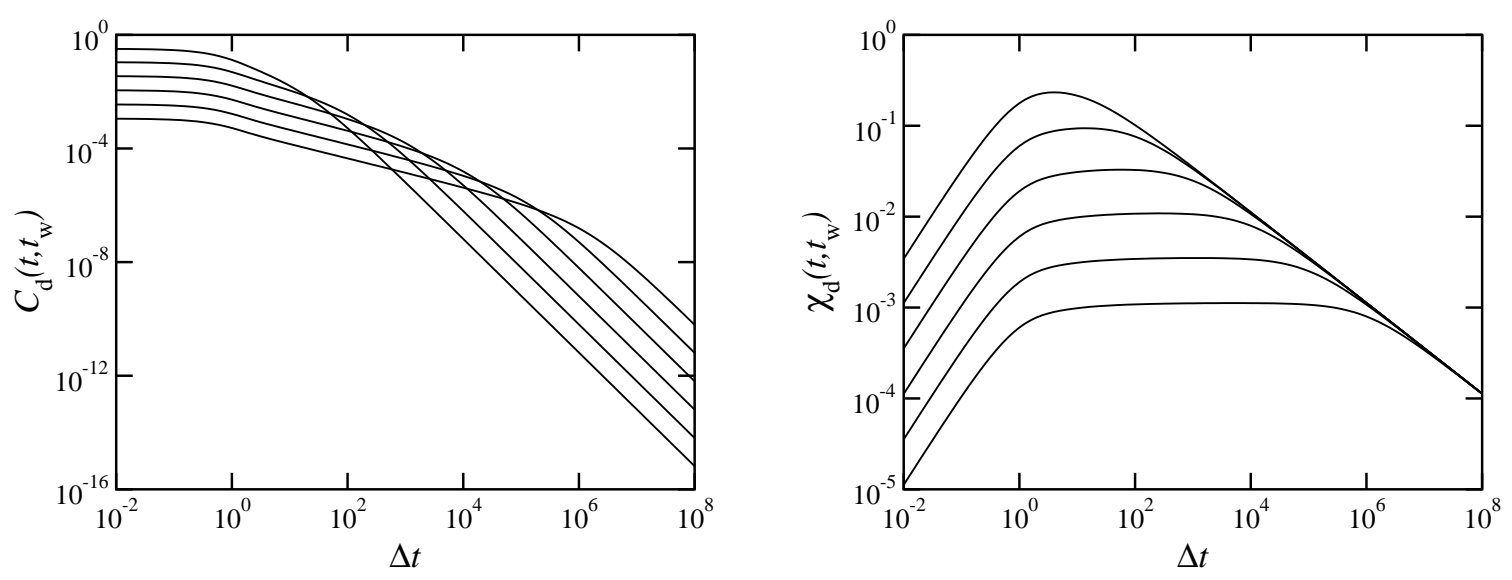

FIG. 3: Defect autocorrelation (left) and susceptibility (right) versus $\Delta t$ for waiting-times $t_{\mathrm{w}}=$ $10^{1}, 10^{2}, \ldots 10^{6}$. Increasing waiting times correspond to decreasing values in the plot for small $\Delta t$.

Now we turn to the dynamics of $C_{\mathrm{d}}\left(t, t_{\mathrm{w}}\right), \chi_{\mathrm{d}}\left(t, t_{\mathrm{w}}\right)$ - examples of which are shown in Fig. 3 as given by (45), (46). The equal-time value of $C_{\mathrm{d}}\left(t_{\mathrm{w}}, t_{\mathrm{w}}\right)=4 c\left(t_{\mathrm{w}}\right)\left(1-c\left(t_{\mathrm{w}}\right)\right) \approx 4 c\left(t_{\mathrm{w}}\right) \approx 2 / \sqrt{\pi t_{\mathrm{w}}}$ for $t_{\mathrm{w}} \gg 1$ decreases with $t_{\mathrm{w}}$, reflecting the decreasing number of particles in the DLPA process (or domain walls in the spin chain). In the regime $\Delta t \ll t_{\mathrm{w}}$ the two-time correlation $C_{\mathrm{d}}\left(t, t_{\mathrm{w}}\right) \approx$ $4 c\left(t_{\mathrm{w}}\right) p_{\mathrm{r}}(\Delta t)$ drops from its initial value due to the random walk motion of the particles around their initial positions at $t_{\mathrm{w}}$. In the aging limit of large $\Delta t$ and $t_{\mathrm{w}}$ one has the expansion $C_{\mathrm{d}}\left(t, t_{\mathrm{w}}\right) \sim$ $2 /\left(\pi \sqrt{t+t_{\mathrm{w}}}\right)\left(1 / \sqrt{t-t_{\mathrm{w}}}-1 / \sqrt{t+t_{\mathrm{w}}}\right)$. This crosses over from $C_{\mathrm{d}}\left(t, t_{\mathrm{w}}\right) \approx 2 /\left(\pi \sqrt{2 \Delta t t_{\mathrm{w}}}\right)$ for $\Delta t \ll$ $t_{\mathrm{w}}$, where it connects smoothly to the initial drop for $\Delta t$ of $\mathcal{O}(1)$ since $p_{\mathrm{r}}(\Delta t) \approx 1 / \sqrt{2 \pi \Delta t}$ for large $\Delta t$, to $C_{\mathrm{d}}\left(t, t_{\mathrm{w}}\right) \approx 2 t_{\mathrm{w}} /\left(\pi \Delta t^{2}\right)$ for $\Delta t \gg t_{\mathrm{w}}$. The integrated response $\chi_{\mathrm{d}}\left(t, t_{\mathrm{w}}\right)$ is non-monotonic in $\Delta t$ and increases on an $\mathcal{O}(1)$ time scale in $\Delta t$ from its initial value $\chi_{\mathrm{d}}\left(t_{\mathrm{w}}, t_{\mathrm{w}}\right)=0$ to a plateau $\chi_{\mathrm{d}}\left(t, t_{\mathrm{w}}\right) \approx 2 / \sqrt{\pi t_{\mathrm{w}}}$ for $\Delta t \ll t_{\mathrm{w}}$ according to $\chi_{\mathrm{d}}\left(t, t_{\mathrm{w}}\right) \approx 4 c\left(t_{\mathrm{w}}\right)\left(1-p_{\mathrm{r}}\left(t-t_{\mathrm{w}}\right)\right)$. This crossover is clear from the spin-chain dynamics: the perturbation associated with $\chi_{\mathrm{d}}\left(t, t_{\mathrm{w}}\right)$ is $\delta H=-h s_{i} s_{i+1}$ which simply increases the coupling between sites $i, i+1$. This enforces alignment of the spins $s_{i}$ and $s_{i+1}$ and hence increases $\left\langle s_{i}(t) s_{i+1}(t)\right\rangle$ on a microscopic time scale. In the aging limit the leading term in the integrated response is just $\chi_{\mathrm{d}}\left(t, t_{\mathrm{w}}\right) \sim 2 / \sqrt{\pi t}$ which connects to the plateau $\chi_{\mathrm{d}}\left(t, t_{\mathrm{w}}\right) \approx 2 / \sqrt{\pi t_{\mathrm{w}}}$ for $\Delta t \ll t_{\mathrm{w}}$ but eventually decreases as $\chi_{\mathrm{d}}\left(t, t_{\mathrm{w}}\right) \approx 2 / \sqrt{\pi \Delta t}$ for $\Delta t \gg t_{\mathrm{w}}$.

For constructing an FD plot (Fig. (4) we are interested in keeping $t$ fixed and varying $t_{\mathrm{w}}$ between 0 and $t$; the functions $C_{\mathrm{d}}\left(t, t_{\mathrm{w}}\right)$ and $\chi_{\mathrm{d}}\left(t, t_{\mathrm{w}}\right)$ are then monotonic in $t_{\mathrm{w}}$. In fact the exact expressions (45), (46) satisfy $C_{\mathrm{d}}(t, t)-C_{\mathrm{d}}\left(t, t_{\mathrm{w}}\right)=\chi_{\mathrm{d}}\left(t, t_{\mathrm{w}}\right)+4\left[c^{2}\left(\left(t+t_{\mathrm{w}}\right) / 2\right)-c^{2}(t)\right]$. Dividing this relation by the equal time value $C_{\mathrm{d}}(t, t)$ yields the relevant normalized quantities

$$
1-\tilde{C}_{\mathrm{d}}\left(t, t_{\mathrm{w}}\right)=\tilde{\chi}_{\mathrm{d}}\left(t, t_{\mathrm{w}}\right)+\frac{c^{2}\left(\frac{t+t_{\mathrm{w}}}{2}\right)-c^{2}(t)}{c(t)(1-c(t))}=\tilde{\chi}_{\mathrm{d}}\left(t, t_{\mathrm{w}}\right)+\mathcal{O}\left(\frac{1}{\sqrt{t}} \frac{t-t_{\mathrm{w}}}{t+t_{\mathrm{w}}}\right) .
$$

In the limit $t \rightarrow \infty$ the extra term in (47) vanishes and we get $1-\tilde{C}_{\mathrm{d}}\left(t, t_{\mathrm{w}}\right)=\tilde{\chi}_{\mathrm{d}}\left(t, t_{\mathrm{w}}\right)$ for all $0 \leq t_{\mathrm{w}} / t \leq 1$. This, however, does not imply that equilibrium FDT holds, i.e. $X_{\mathrm{d}}\left(t, t_{\mathrm{w}}\right)=1$. In fact, working out $\left(\partial / \partial t_{\mathrm{w}}\right) C_{\mathrm{d}}\left(t, t_{\mathrm{w}}\right)$ and $R_{\mathrm{d}}\left(t, t_{\mathrm{w}}\right)$ from (45), (46) and expanding their ratio in the aging limit gives

$$
X_{\mathrm{d}}\left(t, t_{\mathrm{w}}\right) \sim \frac{t_{\mathrm{w}}\left(t+t_{\mathrm{w}}\right)}{t_{\mathrm{w}}\left(t+t_{\mathrm{w}}\right)+\left(t-t_{\mathrm{w}}\right) \sqrt{t^{2}-t_{\mathrm{w}}^{2}}} .
$$

The FDR (48) is a function of the time ratio $t_{\mathrm{w}} / t$ and crosses over from $X_{\mathrm{d}}\left(t, t_{\mathrm{w}}\right)=1$ for $t_{\mathrm{w}} / t \rightarrow 1$ to

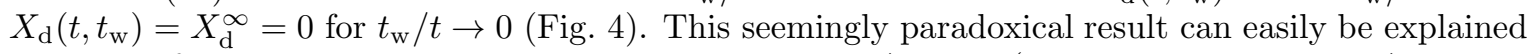
in terms of the expansions given above. In the regime $\Delta t \ll t$ (which is equivalent to $\Delta t \ll t_{\mathrm{w}}$, as considered before) we have, up to subleading corrections for $t \rightarrow \infty, C_{\mathrm{d}}\left(t, t_{\mathrm{w}}\right) \approx 4 c(t) p_{\mathrm{r}}\left(t-t_{\mathrm{w}}\right)$ and $\chi_{\mathrm{d}}\left(t, t_{\mathrm{w}}\right) \approx 4 c(t)\left(1-p_{\mathrm{r}}\left(t-t_{\mathrm{w}}\right)\right)$. So equilibrium FDT indeed holds in this regime and the 

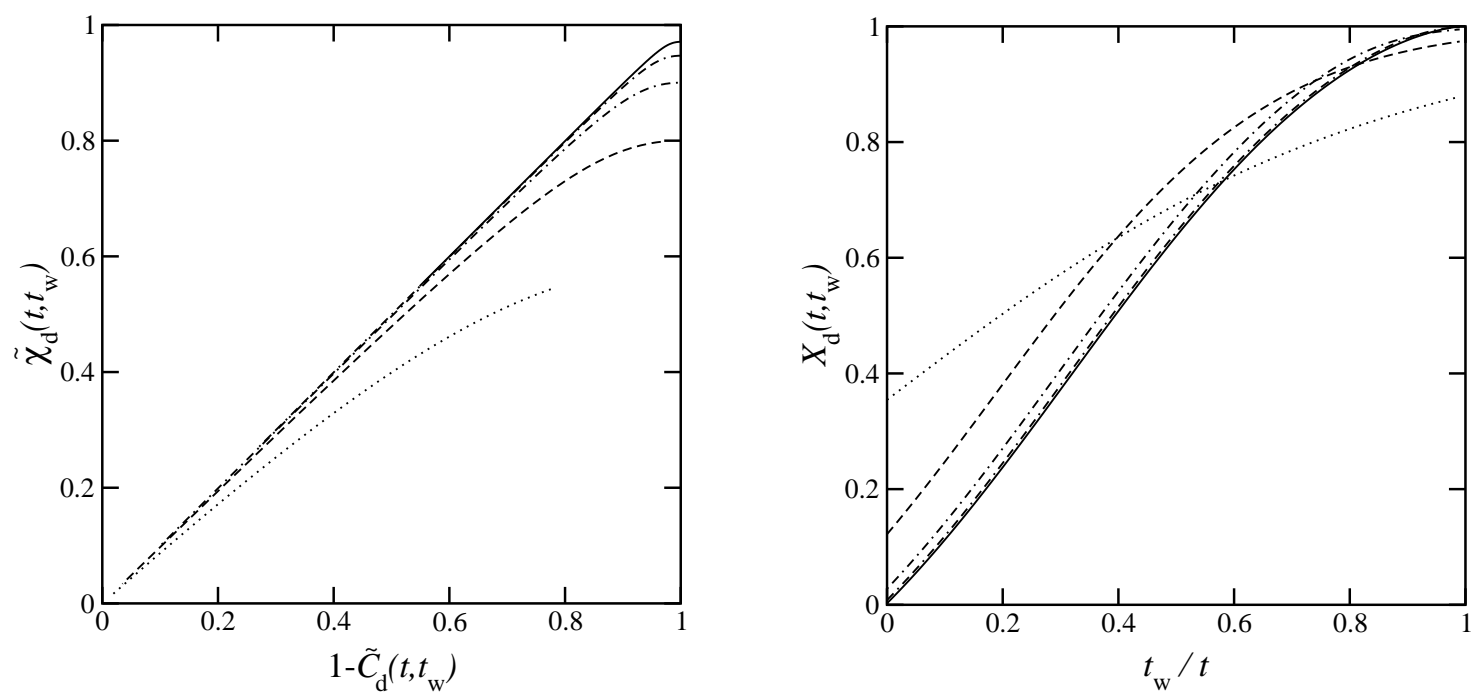

FIG. 4: Normalized FD-plot (left) and the corresponding FDR versus $t_{\mathrm{w}} / t$ (right) for the defect autocorrelation and susceptibility. In both plots $t$ is kept fixed, giving a one-to-one correspondence of the curves, and varies over the range $t=10^{0}$ (dotted), $10^{1 / 2}, 10^{1}, 10^{3 / 2}, 10^{2}$ (solid). The curves of $X_{\mathrm{d}}\left(t, t_{\mathrm{w}}\right)$ for $t=10^{3 / 2}, 10^{2}$ are almost indistinguishable and very close to the limit curve (48).

DLPA process is, to leading order, just an ensemble of independent random walks. Now recall that $p_{\mathrm{r}}(\Delta t) \approx 1 / \sqrt{2 \pi \Delta t}$ for $\Delta t \gg 1$. So at the point where this approximation breaks down, $\Delta t \approx t$, the value of, e.g., $C_{\mathrm{d}}\left(t, t_{\mathrm{w}}\right)$ decreases to an arbitrary small fraction of $C_{\mathrm{d}}(t, t)$ as $t$ increases. This leads to a straight line segment which eventually covers the whole of the (normalized) FD plot while the size of the non-trivial region shrinks as $1 / \sqrt{t}$. In the latter part, for $\tilde{C}_{\mathrm{d}}\left(t, t_{\mathrm{w}}\right) \ll 1 / \sqrt{\pi t}$, one has from the aging expansions of $C_{\mathrm{d}}\left(t, t_{\mathrm{w}}\right)$ and $\chi_{\mathrm{d}}\left(t, t_{\mathrm{w}}\right)$

$$
1-\tilde{\chi}_{\mathrm{d}}\left(t, t_{\mathrm{w}}\right) \approx \frac{1}{\sqrt{\pi t}}+\frac{\sqrt{\pi t}}{2} \tilde{C}_{\mathrm{d}}^{2}\left(t, t_{\mathrm{w}}\right)
$$

Hence the FD plot indeed turns horizontal as $\tilde{C}_{\mathrm{d}}\left(t, t_{\mathrm{w}}\right)$ approaches zero, consistent with (48). In summary, an FD plot is not the appropriate representation for the FDT violation measured by the defect autocorrelation and response. A plot of the FDR as a function of $t_{\mathrm{w}} / t$, however, converges to the non-trivial limit curve given by (48) as times diverge, see Fig. 4

\section{Uniform field: Coherent functions}

For uniform covariances $q_{n}=1$ the defect observable $O_{\mathrm{d}}$ is equivalent to the total energy of the system. According to (23) we have $C_{\mathrm{e}}\left(t, t_{\mathrm{w}}\right)=C\left(0 ; t, t_{\mathrm{w}}\right)$ which may be simplified to give

$$
C_{\mathrm{e}}\left(t, t_{\mathrm{w}}\right)=4 \mathrm{e}^{-2 t}\left[\mathrm{I}_{0}+\mathrm{I}_{1}\right](2 t)-\mathrm{e}^{-2\left(t+t_{\mathrm{w}}\right)}\left[3 \mathrm{I}_{0}+4 \mathrm{I}_{1}+\mathrm{I}_{2}\right]\left(2\left(t+t_{\mathrm{w}}\right)\right) .
$$

This result again has an analog in the associated DLPA process, where it describes the normalized twotime correlation of the total number of particles $\mathcal{N}, C_{\mathrm{e}}\left(t, t_{\mathrm{w}}\right)=(4 / N)\left[\left\langle\mathcal{N}(t) \mathcal{N}\left(t_{\mathrm{w}}\right)\right\rangle-\langle\mathcal{N}(t)\rangle\left\langle\mathcal{N}\left(t_{\mathrm{w}}\right)\right\rangle\right]$. A result similar to (50) was given in [4], for initial conditions corresponding formally to equilibrium at inverse temperature $1 / T=-\infty$. Up to a factor of 4 which appears to be missing in [44], it coincides with (50) for large $t_{\mathrm{w}}$, where one finds the simple scaling form $C_{\mathrm{e}}\left(t, t_{\mathrm{w}}\right) \sim 4 / \sqrt{\pi}\left(1 / \sqrt{t}-1 / \sqrt{t+t_{\mathrm{w}}}\right)$. At equal times, fluctuations in the energy follow as $C_{\mathrm{e}}\left(t_{\mathrm{w}}, t_{\mathrm{w}}\right) \sim(2-\sqrt{2}) C_{\mathrm{d}}\left(t_{\mathrm{w}}, t_{\mathrm{w}}\right)$. This shows that in $C_{\mathrm{e}}\left(t_{\mathrm{w}}, t_{\mathrm{w}}\right)=\sum_{n} C_{n}\left(t_{\mathrm{w}}, t_{\mathrm{w}}\right)$ the non-local $(n \neq 0)$ terms make a contribution $-(\sqrt{2}-1) C_{\mathrm{d}}\left(t_{\mathrm{w}}, t_{\mathrm{w}}\right)$, of the same order as the local term but with opposite sign. For $\Delta t \ll t_{\mathrm{w}}$ the two-time correlation $C_{\mathrm{e}}\left(t, t_{\mathrm{w}}\right) \approx C_{\mathrm{e}}\left(t_{\mathrm{w}}, t_{\mathrm{w}}\right)$ has a plateau but it decreases as $C_{\mathrm{e}}\left(t, t_{\mathrm{w}}\right) \approx 2 t_{\mathrm{w}} /(\Delta t \sqrt{\pi \Delta t})$ when $\Delta t \gg t_{\mathrm{w}}$.

By setting $k=0$ in the Fourier transform (B4) we find that $\chi_{\mathrm{e}}\left(t, t_{\mathrm{w}}\right)=\chi\left(0 ; t, t_{\mathrm{w}}\right) \equiv 0$ at all times. This is for the simple reason that the perturbation is proportional to the Hamiltonian and therefore just rescales the temperature, which obviously has no effect in the $T \rightarrow 0$ limit considered here. We 
note that $\chi_{\mathrm{e}}\left(t, t_{\mathrm{w}}\right)=\sum_{n} \chi_{n}\left(t, t_{\mathrm{w}}\right)=0$ implies that the sum over all cross-susceptibilities $(n \neq 0)$ exactly balances the local susceptibility $\chi_{\mathrm{d}}\left(t, t_{\mathrm{w}}\right) \equiv \chi_{0}\left(t, t_{\mathrm{w}}\right)$.

An FD-plot for the energy is obviously just a horizontal line and the corresponding FDR is $X_{\mathrm{e}}\left(t, t_{\mathrm{w}}\right)=X_{\mathrm{e}}^{\infty}=X_{\mathrm{d}}^{\infty}=0$. This matches our findings in Sec. IIB in the sense that the FD-plot for the coherent observable is a straight line whose slope is the $X^{\infty}$ of the incoherent observable.

\section{Short-range correlated field}

We have seen above that for local defect observables the FD plot is not appropriate for determining FDT violation effects, since it converges to a straight line in the aging limit. It turns out that the same holds for defect observables defined by short-range correlated fields. To see this, recall from (13) that e.g. the correlation function $C\left(t, t_{\mathrm{w}}\right)$ of the observable is a weighted sum of the non-local defect correlations, and focus on the regime $\Delta t=\mathcal{O}(1)$ that dominates the FD plot for large $t_{\mathrm{w}}$ or $t$. From (20), one then easily shows that whenever a non-local term with given $n \neq 0$ is of the same order as the local contribution $C_{\mathrm{d}}\left(t, t_{\mathrm{w}}\right) \equiv C_{0}\left(t, t_{\mathrm{w}}\right) \sim 2 / \sqrt{\pi t_{\mathrm{w}}} \mathrm{e}^{-\Delta t} \mathrm{I}_{0}(\Delta t)$, it can be written as

$$
C_{n}\left(t, t_{\mathrm{w}}\right) \sim \frac{2}{\sqrt{\pi t_{\mathrm{w}}}} \mathrm{e}^{-\Delta t} \mathrm{I}_{n}(\Delta t)
$$

In the same regime the expression for the non-local susceptibility $\chi_{n}\left(t, t_{\mathrm{w}}\right)$ is identical apart from a minus sign. This shows that, whatever the short-ranged field correlations $q_{n}$, the FD plot of $\chi\left(t, t_{\mathrm{w}}\right)$ vs $C\left(t, t_{\mathrm{w}}\right)$ for the observable considered becomes trivial for long times, just as in the case $q_{n}=\delta_{n, 0}$. We therefore focus on the FDR in the following, which requires analysis of $\left(\partial / \partial t_{\mathrm{w}}\right) C\left(t, t_{\mathrm{w}}\right)$ and the response $R\left(t, t_{\mathrm{w}}\right)=-\left(\partial / \partial t_{\mathrm{w}}\right) \chi\left(t, t_{\mathrm{w}}\right)$ and should become non-trival in the aging limit.

By analogy with the results presented in Sec. IIB3 for spin observables, we will show that the FDR becomes identical to that for the incoherent functions in the aging limit. The procedure is again to prove that the Fourier transforms of the defect functions $\left(\partial / \partial t_{\mathrm{w}}\right) C\left(k ; t, t_{\mathrm{w}}\right)$ and $R\left(k ; t, t_{\mathrm{w}}\right)$ are representations of $\tilde{\delta}$ in the aging-limit and with appropriate normalization.

The expressions (B3) for $C\left(k ; t, t_{\mathrm{w}}\right)$ and the one that follows from (B4) for $R\left(k ; t, t_{\mathrm{w}}\right)$ are rather complicated and it is a priori not clear how they behave as times diverge. Asymptotic expansions in the aging limit $t, t_{\mathrm{w}} \rightarrow \infty$ with $\epsilon \leq t_{\mathrm{w}} / t \leq 1-\delta$ fixed $(\epsilon, \delta>0)$ and $|k| \leq K$ where $K=c / \sqrt{t_{\mathrm{w}}}(c>0$ arbitrarily large but finite), however, capture the relevant features of $C\left(k ; t, t_{\mathrm{w}}\right), R\left(k ; t, t_{\mathrm{w}}\right)$ and have a considerably simpler form:

$$
\begin{aligned}
C\left(k ; t, t_{\mathrm{w}}\right) & \sim \frac{4}{\sqrt{\pi}}\left\{\frac{1}{\sqrt{t}} \mathrm{e}^{-k^{2}\left(t^{2}-t_{\mathrm{w}}^{2}\right) /(4 t)}-\frac{1}{\sqrt{t+t_{\mathrm{w}}}} \mathrm{e}^{-k^{2}\left(t+t_{\mathrm{w}}\right) / 4}\right\} \\
& +2 k \mathrm{e}^{-k^{2}\left(t+t_{\mathrm{w}}\right) / 2}\left\{\operatorname{Erfi}\left(k \frac{t+t_{\mathrm{w}}}{2 \sqrt{t}}\right)-\operatorname{Erfi}\left(k \frac{\sqrt{t+t_{\mathrm{w}}}}{2}\right)\right\}, \\
R\left(k ; t, t_{\mathrm{w}}\right) & \sim \frac{1}{\sqrt{\pi t}}\left(\frac{t_{\mathrm{w}}}{t}\right) k^{2} \mathrm{e}^{-k^{2}\left(t^{2}-t_{\mathrm{w}}^{2}\right) /(4 t)} .
\end{aligned}
$$

$\operatorname{Erfi}(x)$ is the error function with imaginary argument: $\operatorname{Erfi}(x)=(1 / i) \operatorname{Erf}(i x)$ [40]. Note that the arguments of all exponentials and the Erfi's are of $\mathcal{O}(1)$ if $t_{\mathrm{w}} / t$ and $k$ are in the specified range. For $|k|$ larger than $\mathcal{O}\left(1 / \sqrt{t_{\mathrm{w}}}\right)$ the results (52), (53) do not apply.

In (52) the growth of $\operatorname{Erfi}(x) \sim \mathrm{e}^{x^{2}} /(\sqrt{\pi} x)$ is over-compensated by the exponential prefactor and so we can make $C\left(K ; t, t_{\mathrm{w}}\right)$ arbitrarily small by increasing $c$. For larger $k,|k|>K$, the values of $C\left(k ; t, t_{\mathrm{w}}\right)$ as given by (B3) also turn out to be insignificant. Therefore $C\left(k ; t, t_{\mathrm{w}}\right)$ develops an infinitely sharp peak of width $\mathcal{O}\left(1 / \sqrt{t_{\mathrm{w}}}\right)$ at $k=0$ in the aging limit and becomes a realization of $\tilde{\delta}(k)$ when normalized by $C_{\mathrm{d}}\left(t, t_{\mathrm{w}}\right)$, in analogy with (33). Differentiating (152) w.r.t. $t_{\mathrm{w}}$ turns out to reproduce the rigorous expansion for $\left(\partial / \partial t_{\mathrm{w}}\right) C\left(k ; t, t_{\mathrm{w}}\right)$ and similar arguments apply. Hence $C\left(t, t_{\mathrm{w}}\right) \sim N_{\mathrm{c}} C_{\mathrm{d}}\left(t, t_{\mathrm{w}}\right)$ and $\left(\partial / \partial t_{\mathrm{w}}\right) C\left(t, t_{\mathrm{w}}\right) \sim N_{\mathrm{c}}\left(\partial / \partial t_{\mathrm{w}}\right) C_{\mathrm{d}}\left(t, t_{\mathrm{w}}\right)$ for any short-range correlated field with $N_{\mathrm{c}} \neq 0$.

The expansion (53) for the response function $R\left(k ; t, t_{\mathrm{w}}\right)$ also peaks sharply in the region $|k| \leq K$ near $k=0$; it follows from (B4) that outside this $k$-range $R\left(k ; t, t_{\mathrm{w}}\right)$ is insignificantly small again. We have, however, $R\left(0 ; t, t_{\mathrm{w}}\right)=0$ at all times. Nevertheless, $R_{\mathrm{d}}\left(t, t_{\mathrm{w}}\right)$ yields normalization and the ratio of both vanishes in the aging-limit for any $k \neq 0$ (modulo $2 \pi$ ). So $R\left(k ; t, t_{\mathrm{w}}\right.$ ) becomes a realization of $\tilde{\delta}(k)$ when normalized by $R_{\mathrm{d}}\left(t, t_{\mathrm{w}}\right)$, i.e. two infinitely sharp peaks at $0^{+}$and $0^{-}$. Therefore $R\left(t, t_{\mathrm{w}}\right) \sim N_{\mathrm{c}} R_{\mathrm{d}}\left(t, t_{\mathrm{w}}\right)$ for any short-range correlated field with $N_{\mathrm{c}} \neq 0$. 
Since $R\left(t, t_{\mathrm{w}}\right) \sim N_{\mathrm{c}} R_{\mathrm{d}}\left(t, t_{\mathrm{w}}\right)$ and $\left(\partial / \partial t_{\mathrm{w}}\right) C\left(t, t_{\mathrm{w}}\right) \sim N_{\mathrm{c}}\left(\partial / \partial t_{\mathrm{w}}\right) C_{\mathrm{d}}\left(t, t_{\mathrm{w}}\right)$, any defect observable $O_{\mathrm{d}}$ with short-range correlated fields $\epsilon_{i}$ and $N_{\mathrm{c}} \neq 0$ ultimately gives the same FDR as the incoherent functions. The scaling of the peaks in (52), (53) implies associated time-dependent length scales in real space. As in the spin observable case the FDR will thus display a crossover (see Fig. 5) when these length scales become comparable with the length over which the fields $\epsilon_{i}$ are correlated. We note finally that, in contrast to the response $R_{n}\left(t, t_{\mathrm{w}}\right)$ discussed above, the integrated response or susceptibility $\chi_{n}\left(t, t_{\mathrm{w}}\right)$ displays somewhat unusual behaviour; e.g. the local value $\chi_{0}\left(t, t_{\mathrm{w}}\right)$ dominates the non-local terms for all times, so that to leading order there is no real-space length scale associated with the defect susceptibility. One also finds non-trivial features in the FD plots and FDRs for the cross-correlations $C_{n}\left(t, t_{\mathrm{w}}\right)$ and susceptibilities $\chi_{n}\left(t, t_{\mathrm{w}}\right)$ [4]. However, in the aging-limit and for any fixed $n \neq 0$ the FDR for the local observable $(n=0)$ is recovered as in the spin observable case.

\section{Infinite-range correlated field}

We next consider the FDR for observables defined by infinite-range correlated fields. As for shortrange correlated fields, we will not discuss the integrated quantities $C\left(t, t_{\mathrm{w}}\right)$ and $\chi\left(t, t_{\mathrm{w}}\right)$ in detail. One finds again that these give a trivial FD plot for long times, although the argument for this is somewhat more subtle than in (51) because one needs to consider an infinite range of distances $n$.

The exact expressions for the two-time correlation functions and susceptibilities for defect observables with power-law covariances follow from (23) by substitution of $C\left(k ; t, t_{\mathrm{w}}\right)$, Eq. (B3), $\chi\left(k ; t, t_{\mathrm{w}}\right)$, Eq. (B4), and $q_{\mathrm{P}}(k)$, Eq. (25). The response $R\left(t, t_{\mathrm{w}}\right)$ is then obtained from $\chi\left(t, t_{\mathrm{w}}\right)$ by $R\left(t, t_{\mathrm{w}}\right)=-\left(\partial / \partial t_{\mathrm{w}}\right) \chi\left(t, t_{\mathrm{w}}\right)$ as usual. The resulting equations are rather bulky and too complex for a meaningful discussion. So we immediately turn to the aging limit, where we can use the following, asymptotically exact, approximations. Firstly we replace the exact expressions in (23) for $C\left(k ; t, t_{\mathrm{w}}\right)$, $R\left(k ; t, t_{\mathrm{w}}\right)$ by (52), (53). Although (52), (53) do not hold outside the range $k \in[-K,+K]$, the contributions to the $k$-integrals are subleading. Secondly, as the integrands have infinitely sharp peaks at $k=0$ in the aging limit, we may replace $q_{\mathrm{P}}(k)$ by the leading term of its expansion at $k=0$, i.e. replace $\sin (k / 2)$ by $k / 2$ in (25). This in turn allows us to extend the limits of integration in (23) from $-\pi,+\pi$ to $-\infty,+\infty$, whereby we again just accumulate subleading errors. Having made these approximations, which still yield asymptotically exact results, the $k$ integrations can be evaluated and we get

$$
\begin{aligned}
& C_{\mathrm{P}}\left(t, t_{\mathrm{w}}\right) \sim \frac{2}{\pi} \Gamma\left(\frac{1+\alpha}{2}\right)\left\{t^{\frac{\alpha-1}{2}}\left(t^{2}-t_{\mathrm{w}}^{2}\right)^{-\frac{\alpha}{2}}-\left(t+t_{\mathrm{w}}\right)^{-\frac{1+\alpha}{2}} \mathrm{~F}\left(\alpha ; \frac{t+t_{\mathrm{w}}}{2 t}\right)\right\}, \\
& R_{\mathrm{P}}\left(t, t_{\mathrm{w}}\right) \sim \frac{2}{\pi} \alpha \Gamma\left(\frac{1+\alpha}{2}\right) t_{\mathrm{w}} t^{\frac{\alpha-1}{2}}\left(t^{2}-t_{\mathrm{w}}^{2}\right)^{-\left(1+\frac{\alpha}{2}\right)},
\end{aligned}
$$

where we have introduced the short-hand

$$
\mathrm{F}\left(\alpha ; \frac{t+t_{\mathrm{w}}}{2 t}\right)=1-(1-\alpha) 2^{-\frac{1+\alpha}{2}}\left(\mathrm{~B}\left(\frac{1}{2}, 1-\frac{\alpha}{2} ; \frac{t+t_{\mathrm{w}}}{2 t}\right)-\mathrm{B}\left(\frac{1}{2}, 1-\frac{\alpha}{2} ; \frac{1}{2}\right)\right) .
$$

In the limit $\alpha \rightarrow 1$, Eqs. (54), (55) reduce to the asymptotic expansions of the incoherent functions $C_{\mathrm{d}}\left(t, t_{\mathrm{w}}\right), R_{\mathrm{d}}\left(t, t_{\mathrm{w}}\right)$ while $\alpha \rightarrow 0$ gives the asymptotic expansions of the coherent ones, i.e. $C_{\mathrm{e}}\left(t, t_{\mathrm{w}}\right)$ from (54) and $R_{\mathrm{e}}\left(t, t_{\mathrm{w}}\right)=0$ from (55). So the power-law covariances (25) again allow us to interpolate between local and global observables. For intermediate exponents $0<\alpha<1$ the two-time correlations in $O_{\mathrm{d}}$ decrease as $t_{\mathrm{w}}^{-1 / 2} \Delta t^{-\alpha / 2}$ in the regime $1 \ll \Delta t \ll t_{\mathrm{w}}$ and cross over to $t_{\mathrm{w}} \Delta t^{-(3+\alpha) / 2}$ for $1 \ll t_{\mathrm{w}} \ll \Delta t$. The response $R_{\mathrm{P}}\left(t, t_{\mathrm{w}}\right)$ behaves as $t_{\mathrm{w}}^{-1 / 2} \Delta t^{-(2+\alpha) / 2}$ for $1 \ll \Delta t \ll t_{\mathrm{w}}$ and $t_{\mathrm{w}} \Delta t^{-(5+\alpha) / 2}$ for $1 \ll t_{\mathrm{w}} \ll \Delta t$. An aging expansion for the FDR again gives non-trivial curves. The derivative $\left(\partial / \partial t_{\mathrm{w}}\right) C_{\mathrm{P}}\left(t, t_{\mathrm{w}}\right)$ follows correctly by differentiating the expansion (54) which, together with (55) yields

$$
X_{\mathrm{P}}\left(t, t_{\mathrm{w}}\right) \sim\left\{1+\frac{t-t_{\mathrm{w}}}{t_{\mathrm{w}}}\left[\frac{1-\alpha}{2 \alpha}+\frac{1+\alpha}{2 \alpha} \sqrt{\frac{t}{t+t_{\mathrm{w}}}}\left(\frac{t-t_{\mathrm{w}}}{t}\right)^{\frac{\alpha}{2}} \mathrm{~F}\left(\alpha ; \frac{t+t_{\mathrm{w}}}{2 t}\right)\right]\right\}^{-1}
$$

The FDR $X_{\mathrm{P}}\left(t, t_{\mathrm{w}}\right)$ is a function of $t_{\mathrm{w}} / t$ only and interpolates between the FDR (48) for the local defect observables $(\alpha \rightarrow 1)$ and $X_{\mathrm{e}}\left(t, t_{\mathrm{w}}\right)=0$ for the energy $(\alpha \rightarrow 0)$. For any power $0<\alpha<1$ (57) crosses over from $X_{\mathrm{P}}\left(t, t_{\mathrm{w}}\right)=1$ for $\Delta t \ll t_{\mathrm{w}}$ to $X_{\mathrm{P}}\left(t, t_{\mathrm{w}}\right)=X_{\mathrm{P}}^{\infty}=0$ for $\Delta t \gg t_{\mathrm{w}}$ (see Fig. [5). 

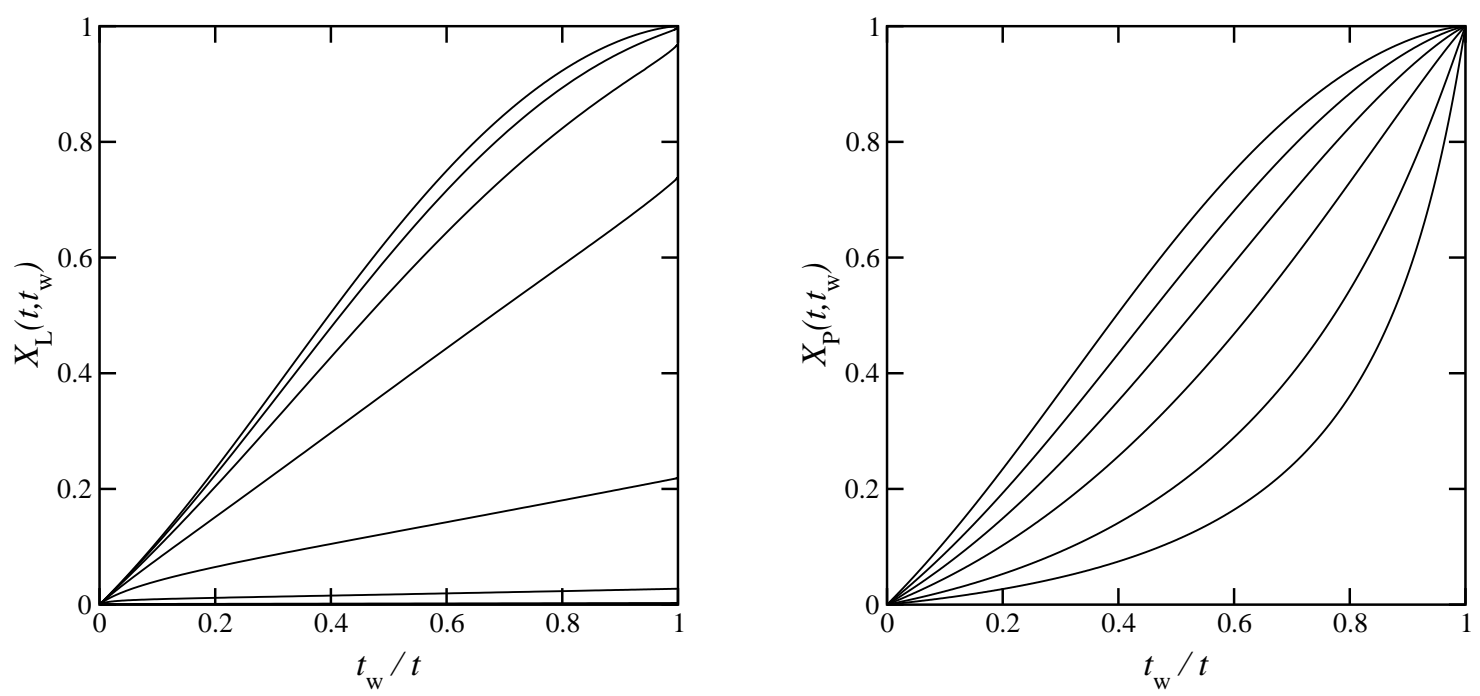

FIG. 5: Left: Time evolution of the defect-observable FDR for a random field with Lorentzian covariances (24) and $a=10^{2}$, correlated over $N_{c}=a \pi \operatorname{coth} a \pi \approx a \pi$ sites. For each curve $t_{\mathrm{w}}$ is kept fixed, varying over the range $t_{\mathrm{w}}=10^{0}, \ldots 10^{6}, \infty$ from bottom to top. The curves for $t_{\mathrm{w}}=10^{0}, 10^{1}, 10^{2}$ are flat; in this regime the observable is effectively identical to the energy. For $t_{\mathrm{w}}=10^{3}, 10^{4}, 10^{5}, 10^{6}$ we see the crossover to the limit curve for $t_{\mathrm{w}} \rightarrow \infty$ given by (48) and corresponding to the incoherent observable. Right: Limit curves of the FDR versus $t_{\mathrm{w}} / t$ for $t, t_{\mathrm{w}} \rightarrow \infty$. From bottom to top these correspond to power-law covariances with exponent $\alpha=0.1,0.2,0.4,0.6,0.8$. The top curve shows the random-field limit $\alpha \rightarrow 1$ for power-laws, and also applies to any short-range correlated field Sec. IIC3 with $N_{\mathrm{c}} \neq 0$ or, in the extreme case, the incoherent functions of Sec. IIC1

\section{Harmonically correlated fields and $X^{\infty}$}

In contrast to spin observables it appears that for defect observables $O_{\mathrm{d}}$ we generically find $X^{\infty}=0$. To prove this claim we may again follow the approach presented in Sec.[IB 5 Introducing an FDR for defect Fourier modes $X\left(k ; t, t_{\mathrm{w}}\right)$ according to (40) based on the two-time defect correlation function (B3) and susceptibility (B34) allows us to write the FDR for any defect observable in the form (41). The full expression for $X\left(k ; t, t_{\mathrm{w}}\right)$ is rather complicated and, in contrast to (42), retains a nontrivial dependence on $k, t$ and $t_{\mathrm{w}}$. The only general features are $X\left(0 ; t, t_{\mathrm{w}}\right)=0$, since $X\left(0 ; t, t_{\mathrm{w}}\right)=$ $X_{\mathrm{e}}\left(t, t_{\mathrm{w}}\right)=0$, and $X\left( \pm \pi ; t, t_{\mathrm{w}}\right)=1+\mathcal{O}\left(\sqrt{t_{\mathrm{w}}} \mathrm{e}^{-4 t_{\mathrm{w}}}\right)$ being independent of $t$ and close to one for $t_{\mathrm{w}} \gg 1$. For intermediate values $0<|k|<\pi$ the $\mathrm{FDR} X\left(k ; t, t_{\mathrm{w}}\right)$ can in fact take arbitrarily large values for appropriate $t_{\mathrm{w}}$ and $t$. To repeat the argument of Sec. IIB5 however, we just have to be able to take the limit $t \rightarrow \infty$ for fixed and finite $t_{\mathrm{w}}$. A scaling analysis of $\left(\partial / \partial t_{\mathrm{w}}\right) C\left(k ; t, t_{\mathrm{w}}\right)$ as obtained from (B3) shows that this quantity develops an infinitely sharp peak at $k=0$. Hence the normalized distribution of $q(k)\left(\partial / \partial t_{\mathrm{w}}\right) C\left(k ; t, t_{\mathrm{w}}\right)$ over $-\pi \leq k \leq \pi$ becomes, for sufficiently well behaved functions $q(k)$, a realization of $\tilde{\delta}(k)$ as $t \rightarrow \infty$. Remarkably, the FDR for defects $X\left(k ; t, t_{\mathrm{w}}\right)$ approaches a simple, smooth function on $\pi<k<\pi$ in the same limit

$$
\lim _{t \rightarrow \infty} X\left(k ; t, t_{\mathrm{w}}\right)=2 \sin ^{2} \frac{k}{4}\left(1+4 t_{\mathrm{w}} \cos ^{2} \frac{k}{4}\right)\left(2-\mathrm{e}^{-4 t_{\mathrm{w}} \sin ^{2} \frac{k}{4}}\right)^{-1} .
$$

Together, these two facts imply that out of the spectrum of FDRs $X\left(k ; t, t_{\mathrm{w}}\right)$ for Fourier modes $k$, the long time limit $t \rightarrow \infty$ again selects the contributions associated with infinite length scales $(k=0)$. These are, in the limit, given by (58) and equal to zero. The FDR (41) for defect observables $X\left(t, t_{\mathrm{w}}\right) \rightarrow 0$ thus vanishes as $t \rightarrow \infty$ regardless of the choice of $q(k)$, except in pathological cases as discussed in Sec. [IB5 Note that because (58) for $k=0$ gives a vanishing result for any $t_{\mathrm{w}}$, one in fact has $\lim _{t \rightarrow \infty} X\left(t, t_{\mathrm{w}}\right)=X^{\infty}=0$, without needing to take $t_{\mathrm{w}} \rightarrow \infty$.

We note finally that the behaviour at short wavelengths is rather more complex for defect observables than for spins. In particular, even for what one might expect to be 'equilibrated' wavelengths, $k^{2} t_{\mathrm{w}} \gg 1$, it is not true that $X\left(k ; t, t_{\mathrm{w}}\right) \approx 1$ for all times $t$, and $X$ deviates significantly from this simple value for large time differences $\Delta t \gg t_{\mathrm{w}}$ as can be seen from (58). 


\section{Physical discussion}

We saw above that apart from pathological exceptions all spin and defect observables give identical values for the asymptotic FDR $X^{\infty}$, with $X^{\infty}=1 / 2$ for spin observables and $X^{\infty}=0$ for defect observables. These slopes are most easily read off from the FDT plots for the coherent observables (magnetization and energy, respectively) which become straight lines in the long-time limit.

It is natural to ask how these results would extend to observables other than those we have considered, such as $O=\sum_{i} \epsilon_{i} s_{i} s_{i+2}$ which involves spin pairs at distance two. We have worked out explicitly the FD properties based on the general solutions given in [38] for the coherent and incoherent versions for this observable [45]; one finds that they are, up to subdominant corrections, identical to those for $O=2 \sum_{i} \epsilon_{i} s_{i} s_{i+1}$. The physical interpretation is simple: $s_{i} s_{i+2}=-1$ if there is exactly one domain wall between spins $i$ and $i+2$, while $s_{i} s_{i+2}=1$ if there is no domain wall or if there are two. The last alternative, however, is suppressed in the aging limit where typical distances between domain walls scale as $\sqrt{t_{\mathrm{w}}}$, and so $s_{i} s_{i+2} \approx s_{i} s_{i+1}+s_{i+1} s_{i+2}-1$. For the coherent observable $\left(q_{n}=\left[\epsilon_{i} \epsilon_{i+n}\right]=1\right)$, this directly explains our observation; for the incoherent version $\left(q_{n}=\delta_{n, 0}\right)$ it follows from the fact that the correlations of $s_{i} s_{i+1}$ and $s_{i+1} s_{i+2}$ are identical to the autocorrelations of $s_{i} s_{i+1}$ in the aging limit.

By a similar reasoning we can now predict the FD behaviour of higher-order observables of the form

$$
O_{j}^{(k)}=\sum_{i} \epsilon_{i} \prod_{\eta=1}^{k} s_{i+j_{\eta}},
$$

with $k \geq 3$ and $j_{1}=0 ; j_{2}, \ldots, j_{k}$ specify the relative displacements of the spins in the $k$-th order products. For even $k$, each term $\prod_{\eta} s_{i+j_{\eta}}$ again has a sign depending on the number of domain walls between spins $i$ and $i+j_{k}$. In the aging limit, configurations where more than one domain wall occurs can be neglected, so that we can replace the product by $s_{i} s_{i+j_{k}}$. By the same argument as above, this is essentially equivalent to $j_{k} \times s_{i} s_{i+1}$ and so should again give $X^{\infty}=0$.

For odd $k$, on the other hand, the sign of $\prod_{\eta} s_{i+j_{\eta}}$ is essentially determined by the sign of the domain which the spin $s_{i}$ finds itself in. The leading contribution is now given by configurations with no domain walls between $s_{i}$ and $s_{i+j_{k}}$. Configurations with at least one domain wall are again suppressed in the aging limit. We can therefore replace the product simply by $s_{i}$ to leading order, giving an asymptotic FDR of $X^{\infty}=1 / 2$ as for genuine first order spin observables.

The fact that observables of even and odd order behave in different ways can also be motivated mathematically from the hierarchy of the equations obeyed by the multi-spin correlation functions where the even and odd orders $k$ turn out to decouple completely [38]. This is a peculiarity of the one-dimensional Ising model, whereas in the generic case one would expect all levels of the hierarchy to couple to each other, resulting in a unique value of $X^{\infty}$. We indeed find strong evidence for this in the two-dimensional case below.

From a more physical point of view, the existence of two different values of $X^{\infty}$ could be related to the fact that in the one-dimensional chain at $T=0$ one has both a critical point and an ordered phase. The result $X^{\infty}=0$ for defect observables could thus be related to the ordinary results for coarsening in $d \geq 2$ after a quench to an ordered phase, while $X^{\infty}=1 / 2$ for the spins would reflect the critical aspects of coarsening at $T=T_{c}=0$.

It might be interesting - though rather complicated - to use the methods described above and in Ref. 38] to study higher order observables different from (59) for which the above leading order approximations do not apply, for example, $1-s_{i} s_{i+1}-s_{i+1} s_{i+2}+s_{i} s_{i+2}$, which corresponds to a quadratic operator in bond variables, $4 b_{i} b_{i+1}$. We are currently exploring this issue.

To recap, the central result of this section is that (almost) all observables of the form (59) interpolate between an equilibrium like behaviour with $X=1$ and an asymptotic FDR $X^{\infty}$. The latter are given by the values of $X(k \rightarrow 0)$, as was argued in 33, 34, 35]. We have shown that the most efficient way of extracting $X^{\infty}$ is by studying coherent functions. These results motivate the following section where the $2 d$ Ising model is studied at criticality. 


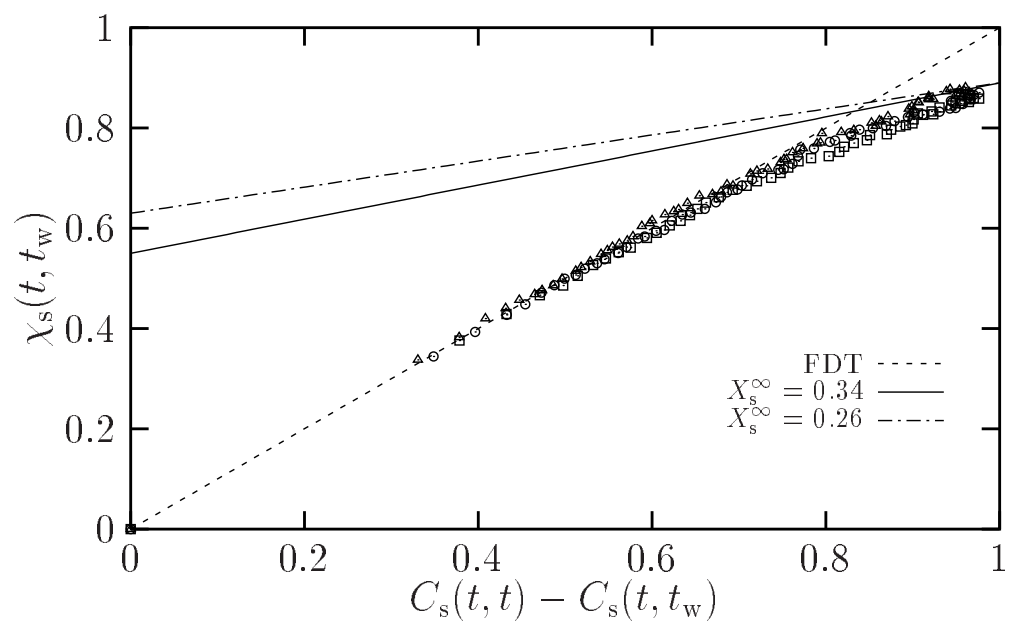

FIG. 6: FD plots for spin autocorrelation and response. Three waiting times, $t_{\mathrm{w}}=43,179$ and 460 are represented by squares, circles and triangles, respectively. The dashed line with slope one shows the equilibrium FDT. The full and dash-dotted lines have slopes $X_{\mathrm{s}}^{\infty}=0.34$ and $X_{\mathrm{s}}^{\infty}=0.26$, respectively; these are discussed in the text.

\section{THE $2 d$ ISING MODEL}

In this section, we report on numerical simulations of the $2 d$ Ising model. It is defined by the Hamiltonian

$$
\mathcal{H}=-\sum_{\langle i, j\rangle} s_{i} s_{j}
$$

where the $s_{i}(i=1, \cdots, N)$ are $N$ Ising spins located on the sites of a square lattice with periodic boundary conditions and linear size $L$; the sum is over nearest neighbor pairs. We perform MonteCarlo simulations using a standard Metropolis algorithm where the spins are randomly updated. One Monte Carlo step represents $N$ attempts to flip a spin.

The system is prepared in a random state, corresponding to an infinite initial temperature. It is then quenched at $t=0$ to the critical temperature $T_{c}=\frac{1}{2} \ln (1+\sqrt{2})$. As stated in Sec. [D] we focus on the four natural FD relations for the Ising model, constructed from the coherent and incoherent dynamical functions of spin and defect observables. The system size we use is different for coherent and incoherent objects. Incoherent objects reflect the behaviour of individual spins or defects, and simulating a very large system is advantageous in that it makes an average over many initial conditions unnecessary. Coherent objects like $C_{\mathrm{e}}\left(t, t_{\mathrm{w}}\right)$ or $C_{\mathrm{m}}\left(t, t_{\mathrm{w}}\right)$, on the other hand, have an amplitude of order $1 / N$. One should thus simulate many initial conditions of the smallest possible system, with the opposite constraint that the system has to be out of equilibrium even for the largest simulated time scale, $t_{\text {simu }}$, giving the condition $\xi\left(t_{\text {simu }}\right) \ll L$. Our results are obtained with $t_{\text {simu }}=10^{5}, L=300$ for coherent functions, and $L=500$ for incoherent ones. Only a few samples over initial conditions are necessary for incoherent correlation functions, while 1000 initial conditions were sampled for coherent ones. This is also the number of realizations necessary to get the four susceptibilities we have computed.

We now describe our results, starting with spin observables and then turn to defect observables.

\section{A. Spin observables}

The two-time scaling behavior of the incoherent spin functions $C_{\mathrm{s}}\left(t, t_{\mathrm{w}}\right)$ and $\chi_{\mathrm{s}}\left(t, t_{\mathrm{w}}\right)$ has been the subject of a number of publications, as described in Sec.[C] We refer to the references cited there and directly present in Fig. [6 the FD plot for the spin autocorrelation and susceptibility. A very similar FD plot has been reported in Ref. [29], although a somewhat different susceptibility $\int_{0}^{t_{\mathrm{w}}} \mathrm{d} \tau R_{\mathrm{s}}(t, \tau)$ 


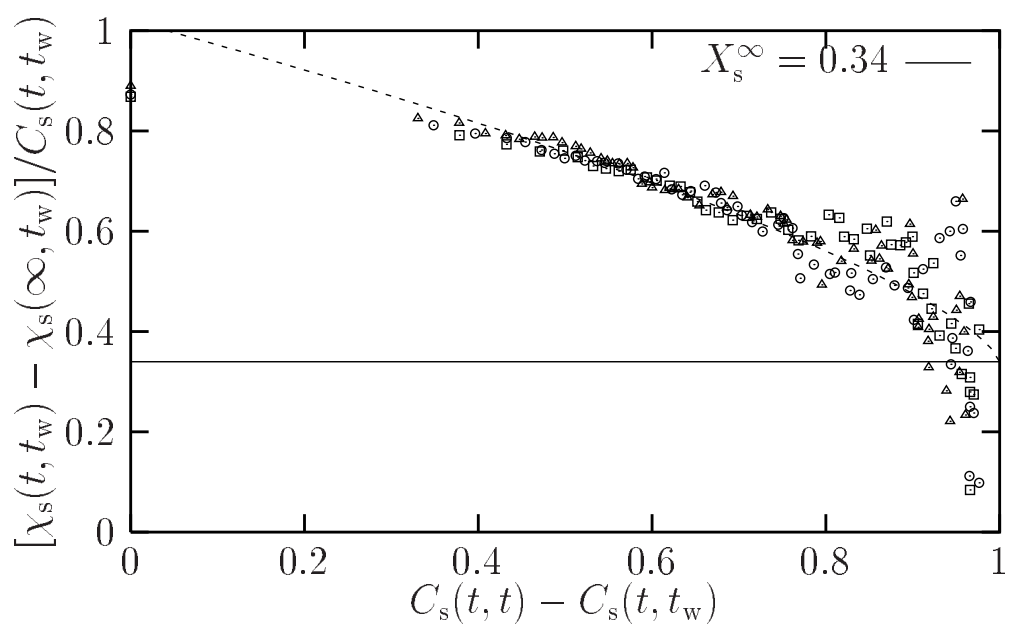

FIG. 7: Tentative extrapolation of the infinite time slope of the FD plots of Fig. 6] The lines are only suggestive, indicating that an asymptotic FDR of $X_{\mathrm{s}}^{\infty}=0.34$ is compatible with the data in the regime $C_{\mathrm{s}}(t, t)-C_{\mathrm{s}}\left(t, t_{\mathrm{w}}\right) \approx 1$. The different symbols have the same meaning as in Fig. [6]

was plotted there, so that the FD plot looks reversed compared to Fig. [6 Otherwise, we find the features anticipated in Sec. IC The FD plot is characterized by an initial part which follows the equilibrium FDT, corresponding to short, equilibrated length scales. For larger time differences, the FD plot deviates from the FDT in a non-trivial manner due to the non-equilibrated fluctuations at small wavevectors. In the limit of large time differences the FD plot has a nonzero slope $X_{\mathrm{s}}^{\infty}$, in contrast to the zero slope obtained below the critical point [7, [8]. These features make the FD plot rather similar to the one obtained in $d=1$, see Fig. 2 (left).

The infinite time value for the slope of the FD plot for the $2 d$ Ising model was estimated in Ref. 29] as $X_{\mathrm{s}}^{\infty}=0.26$. We recognize from Fig. [6] that the crossover from $X=1$ to $X_{\mathrm{s}}^{\infty}<1$ takes place over a very small range of the correlator, and that a precise determination of the infinite-time value of the FDR is difficult. A tentative numerical extrapolation is shown Fig. 7 where the quantity $\left(\chi_{\mathrm{s}}\left(t, t_{\mathrm{w}}\right)-\chi_{\mathrm{s}}\left(\infty, t_{\mathrm{w}}\right)\right) / C_{\mathrm{s}}\left(t, t_{\mathrm{w}}\right)$ is plotted against $C_{\mathrm{s}}(t, t)-C_{\mathrm{s}}\left(t, t_{\mathrm{w}}\right)$; as the abscissa approaches 1 (i.e. for large time differences), the ordinate should converge to $X_{\mathrm{s}}^{\infty}$. The figure shows that the value $X_{\mathrm{s}}^{\infty}=0.34$ is compatible with the data, but even though we use larger waiting times than in Ref. [29] there is substantial scatter in the points. However, we have more precise estimates of $X_{\mathrm{s}}^{\infty}$ to guide us, as we now describe.

The study of the model in $d=1$ in Sec. II showed that the crossover from $X=1$ to $X_{\mathrm{s}}^{\infty}$ for spin functions reflected the different dynamics of large and small wavevectors which for the $d=2$ case would be defined according to $k \xi\left(t_{\mathrm{w}}\right) \lessgtr 1$. The dynamical behaviour of the small wavevectors was governed by the asymptotic FDR $X_{\mathrm{s}}^{\infty}$. This suggests that a much simpler measurement of $X_{\mathrm{s}}^{\infty}$ should be possible by focusing on the $k \rightarrow 0$ limit, i.e. by measuring the correlation and susceptibility of the magnetization density $m(t)$. The resulting FD plot is reported in Fig. 8 As for the $1 d$ case, a very simple result is obtained, with the FD plot extremely well fitted by a simple straight line. The fit holds for several decades of time, $t_{\mathrm{w}}<t<t_{\mathrm{simu}}$, for each waiting time $t_{\mathrm{w}}$ that we have considered, providing strong evidence that $X_{\mathrm{m}}\left(t, t_{\mathrm{w}}\right)=X_{\mathrm{m}}^{\infty}$ at all times. Furthermore, the slopes of the three curves in Fig. 8 are very close to one another, and this allows us to report the value

$$
X_{\mathrm{m}}^{\infty}=0.340 \pm 0.005 .
$$

This is the value we used to fit the data for the incoherent spin functions in Figs. 6 and 7 demonstrating that the data are consistent with the equality $X_{\mathrm{s}}^{\infty}=X_{\mathrm{m}}^{\infty}$. This is somewhat different from the value reported in Ref. [29], but we believe that our measurement from the magnetization is much more reliable than the extrapolation of the incoherent spin functions, as explained above. We note also that this value is in extremely good agreement with the two-loop expansion value reported in Ref. [33]. However, unlike the $1 d$ case, we do not have a simple physical argument to explain the actual numerical value. 


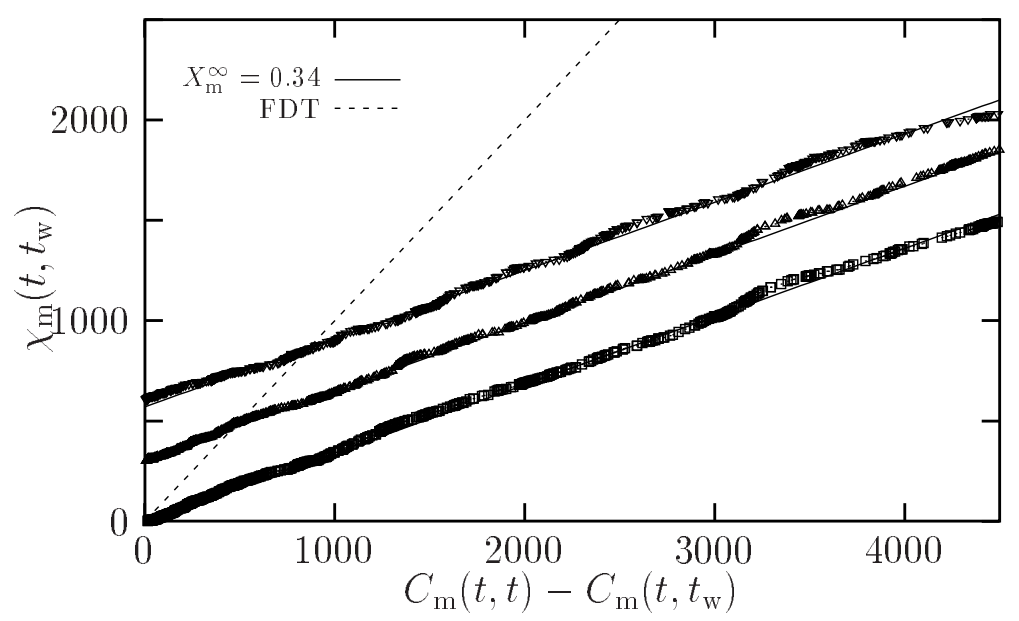

FIG. 8: FD plots of correlation and susceptibility for the magnetization. The three curves are for waiting times $t_{\mathrm{w}}=46,193$ and 720 (bottom to top). The curves for $t_{\mathrm{w}}=193$ and $t_{\mathrm{w}}=720$ have been shifted vertically for clarity, since they would otherwise overlap with the curve for $t_{\mathrm{w}}=46$; the unshifted curves all pass through the origin as they should. The dashed line is the equilibrium FDT. The full lines have slope $X_{\mathrm{m}}^{\infty}=0.34$.

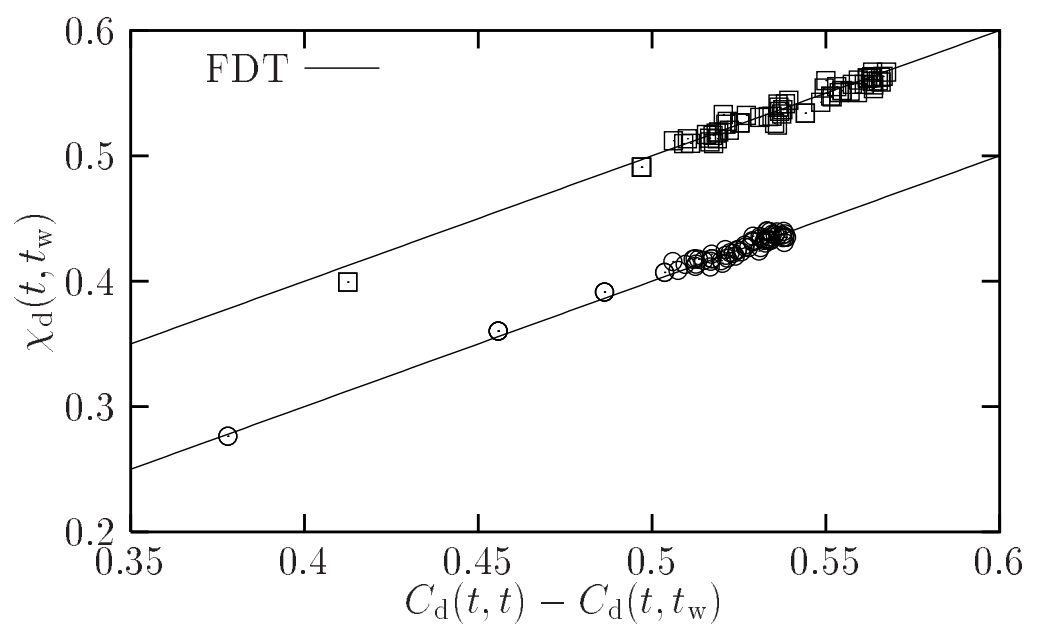

FIG. 9: FD plots for defect autocorrelation and susceptibility. Data for two waiting times, $t_{\mathrm{w}}=43$ (squares) and $t_{\mathrm{w}}=179$ (circles), are presented. The second one has been vertically shifted for clarity. The full lines represent the equilibrium FDT.

\section{B. Defect observables}

We now turn to defect observables. The simplest functions to consider are the defect autocorrelation function and the conjugate susceptibility. These quantities have been studied recently for kinetically constrained Ising models (in particular the Fredrickson-Andersen model in 1d), where they were shown to give rise to simple FD plots [36]. We present the corresponding FD plot for the $2 d$ Ising model in Fig. 9. Again an apparently very simple result is obtained, with the FD plot very well fitted by the equilibrium straight line with $X=1$. This is an unexpected result, since the system is far from equilibrium as was demonstrated by the study of spin observables in the previous section. It could also be taken to imply, as in Ref. [36], that the asymptotic value of the FDR associated with the defects has the equilibrium value $X_{\mathrm{d}}^{\infty}=1$. 


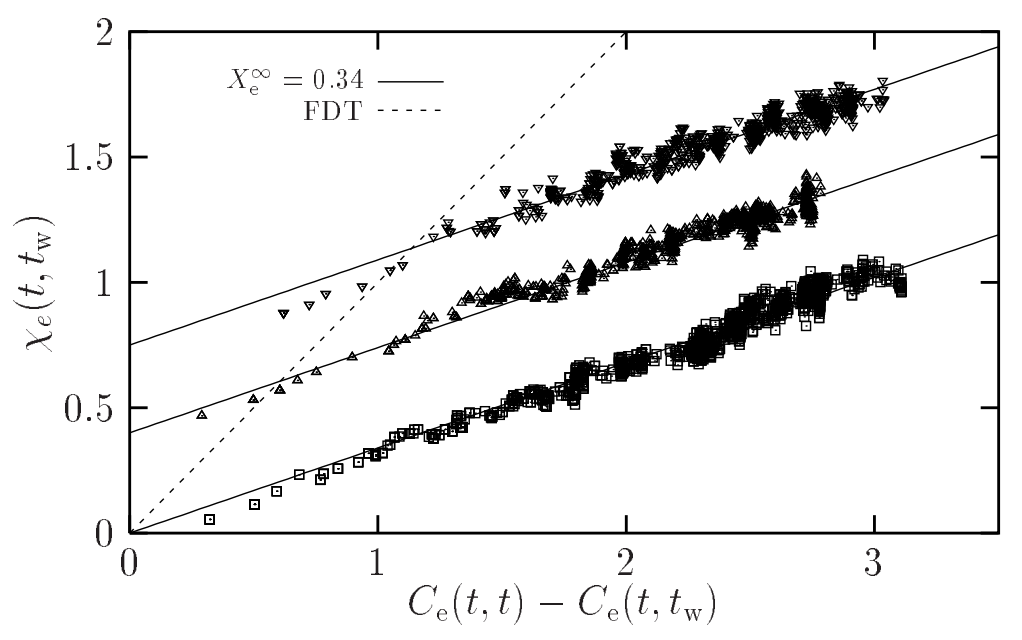

FIG. 10: FD plots for the energy density. The three curves are for $t_{\mathrm{w}}=80,193,464$ (bottom to top). The curves for $t_{\mathrm{w}}=193$ and $t_{\mathrm{w}}=464$ have been vertically shifted for clarity, and would otherwise again pass through the origin. The dashed curve is the equilibrium FDT; the full lines have slope $X_{\mathrm{e}}^{\infty}=0.34$.

Our above study of the $1 d$ model again clarifies the situation. There, we found that the incoherent dynamical functions of the defects exhibited a crossover from equilibrium to non-equilibrium behaviour, but that the non-equilibrium part was barely visible in an FD plot since the crossover occurs when correlators have already decayed to very small values. This suggests that the apparent equilibrium behaviour observed in simulations for the $2 d$ Ising and $1 d$ Fredrickson-Andersen models is simply a good approximation to numerical data, but may miss non-trivial FD relations at large times due to limitations in the numerical analysis. However, as for the spin observables, the solution to this problem is straightforward and consists in focusing on the $k \rightarrow 0$ limit. We thus investigate next the coherent functions for the defects which are the autocorrelation and susceptibility for the energy density.

The resulting FD plot for the energy density is shown in Fig. 10. As for the magnetization, very good fits by pure straight lines are obtained, implying the equality $X_{\mathrm{e}}\left(t, t_{\mathrm{w}}\right)=X_{\mathrm{e}}^{\infty}$. Note, however, that these plots have more noise than the ones for the magnetization. This is due to the fact that the abscissa now involves a genuine connected correlator, in which the nonzero average of the energy density needs to be subtracted off. Nonetheless, the slopes of the FD plots in Fig. 10 are very close to one another and give the result

$$
X_{\mathrm{e}}^{\infty}=0.33 \pm 0.02 .
$$

An important outcome of this paper is that this value is compatible, within error bars, with the value reported above for the asymptotic FDT for the magnetization and the spins. This strongly suggests that the various infinite time FDRs that we have measured in the $2 d$ Ising model are all equal, and that the critical point of the $2 d$ Ising model is described by a single new universal quantity

$$
X_{\mathrm{s}}^{\infty}=X_{\mathrm{m}}^{\infty}=X_{\mathrm{d}}^{\infty}=X_{\mathrm{e}}^{\infty} \equiv X^{\infty} \approx 0.340 .
$$

\section{SUMMARY AND DISCUSSION}

In this paper we have studied the relation between two-time, multi-spin, correlation and response functions in the non-equilibrium critical dynamics of Ising models, analytically in the $d=1$ case, and numerically in $d=2$. We have shown that FDRs, while observable-dependent, fall into welldefined classes, which are qualitatively similar to those observed in various glassy systems. All FDT violations can be understood by considering separately the contributions from large wavevectors, which are at quasi-equilibrium and obey FDT, and from small wavevectors where a generalized FDT holds with a non-trivial fluctuation-dissipation ratio $X^{\infty}=X(k \rightarrow 0)$. In $d=1$, we find through exact calculations $X^{\infty}=\frac{1}{2}$ for spin observables and $X^{\infty}=0$ for defect observables. In $d=2$ we find 
numerically a unique $X^{\infty} \simeq 0.34$ for all observables. These results suggest that the definition of an effective temperature $T_{\text {eff }}=T / X^{\infty}$ for large length scales is generically possible in non-equilibrium critical dynamics.

Further, this work also suggests many interesting lines for future investigation. An important question is what are the limiting FDRs in diffusive models that are analogous to the $1 d$ Ising model but have glassy features, for example the one-spin facilitated Fredrickson-Andersen model [47] or symmetric plaquette models [36]. Also, it would be interesting to confirm our results for the $2 d$ Ising model by analyzing higher order correlation functions by means of the RG techniques used in Ref. [33, 34, 35] to confirm the uniqueness of the FDR. This would make this function an interesting quantity to study in more generic non-equilibrium situations such as driven interfaces or driven diffusive systems.

\section{Acknowledgments}

We acknowledge financial support from Österreichische Akademie der Wissenschaften and EPSRC Grant No. 00800822 (PM), Marie Curie Fellowship No HPMF-CT-2002-01927, CNRS and Worcester College, Oxford (LB), EPSRC Grant No. GR/R83712/01 (LB and JPG), the Glasstone Fund (JPG), and Nuffield Grant No. NAL/00361/G (PS). Numerical results were obtained on OSWELL at the Oxford Supercomputing Center, Oxford University, UK

\section{APPENDIX A: MODIFIED BESSEL FUNCTIONS}

Here we briefly summarize the main properties of modified Bessel functions $\mathrm{I}_{n}(x)$ that are relevant for the analysis given above. A comprehensive description may be found in [40]. For integer order $n$, $\mathrm{I}_{n}(x)$ has the integral representation

$$
\mathrm{I}_{n}(x)=\int_{0}^{\pi} \frac{\mathrm{d} \varphi}{\pi} \cos n \varphi \mathrm{e}^{x \cos \varphi}
$$

from which the functional relations

$$
\frac{\partial}{\partial x} \mathrm{I}_{n}(x)=\frac{1}{2}\left[\mathrm{I}_{n-1}+\mathrm{I}_{n+1}\right](x) \text { and } \frac{2 n}{x} \mathrm{I}_{n}(x)=\left[\mathrm{I}_{n-1}-\mathrm{I}_{n+1}\right](x)
$$

follow immediately. In particular it is clear from that $\mathrm{I}_{-n}(x)=\mathrm{I}_{n}(x)$ and $\mathrm{I}_{n}(-x)=(-1)^{n} \mathrm{I}_{n}(x)$. The aging expansions of our results are based on the asymptotic formula

$$
\mathrm{I}_{n}(x)=\frac{\mathrm{e}^{x}}{\sqrt{2 \pi x}}\left(1+\frac{1-4 n^{2}}{8 x}+\mathcal{O}\left(\frac{1}{x^{2}}\right)\right),
$$

which applies in the limit of large arguments $x$ for fixed order $n$. For the derivation of the Fourier transforms of multi-spin correlation and response functions we use

$$
\begin{aligned}
\sum_{n} \mathrm{e}^{-i n x} \mathrm{I}_{n}(a) & =\mathrm{e}^{a \cos x} \\
\sum_{n} \mathrm{e}^{-i n k} \mathrm{I}_{n-m}(a) \mathrm{I}_{n+m}(a) & =\mathrm{I}_{2 m}\left(2 a \cos \frac{k}{2}\right), \\
\sum_{n} \mathrm{e}^{-i n k} \mathrm{I}_{n}(a)\left(\mathrm{I}_{n+m}(b)+\mathrm{I}_{n-m}(b)\right) & =2 \mathrm{~T}_{m}\left(\frac{b+a \cos k}{A}\right) \mathrm{I}_{m}(A),
\end{aligned}
$$

where (A5), A6 follow from (A1), the well known identity (A4) and trigonometric relations. In (A6) $0<a \leq b$ is required, $A=\sqrt{a^{2}+b^{2}+2 a b \cos k}$ and the $\mathrm{T}_{n}(x)=\cos (n$ arccos $x)$ are Chebyshev polynomials of degree $n$ in $x$. 


\section{APPENDIX B: FOURIER TRANSFORMS}

The Fourier transforms of spin correlation and response functions (18), (19) follow immediately when using (A4):

$$
\begin{aligned}
& C\left(k ; t, t_{\mathrm{w}}\right)=\mathrm{e}^{-\left(t+t_{\mathrm{w}}\right)(1-\cos k)}\left\{1+\int_{0}^{2 t_{\mathrm{w}}} \mathrm{d} \tau \mathrm{e}^{-\tau \cos k}\left[\mathrm{I}_{0}+\mathrm{I}_{1}\right](\tau)\right\} \\
& \chi\left(k ; t, t_{\mathrm{w}}\right)=\frac{1}{2} \int_{t_{\mathrm{w}}}^{t} \mathrm{~d} \tau \mathrm{e}^{-(t-\tau)(1-\cos k)} \mathrm{e}^{-2 \tau}\left[\mathrm{I}_{0}+2 \mathrm{I}_{1}+\mathrm{I}_{2}\right](2 \tau)
\end{aligned}
$$

For defect correlations, however, a direct transformation of (20) yields a rather intractable expression. Therefore we first rewrite (20) using the identity (which can be verified by differentiation)

$$
\mathrm{e}^{-\left(t-t_{\mathrm{w}}\right)} \mathrm{I}_{n}\left(t-t_{\mathrm{w}}\right)=\mathrm{e}^{-\left(t+t_{\mathrm{w}}\right)} \mathrm{I}_{n}\left(t+t_{\mathrm{w}}\right)-\int_{t-t_{\mathrm{w}}}^{t+t_{\mathrm{w}}} \mathrm{d} \tau \frac{1}{2} \mathrm{e}^{-\tau}\left[\mathrm{I}_{n-1}-2 \mathrm{I}_{n}+\mathrm{I}_{n+1}\right](\tau)
$$

as

$$
\begin{gathered}
C_{n}\left(t, t_{\mathrm{w}}\right)=\mathrm{e}^{-2\left(t+t_{\mathrm{w}}\right)}\left[\mathrm{I}_{n}^{2}-\mathrm{I}_{n-1} \mathrm{I}_{n+1}\right]\left(t+t_{\mathrm{w}}\right)+\frac{1}{2} \int_{t-t_{\mathrm{w}}}^{t+t_{\mathrm{w}}} \mathrm{d} \tau \mathrm{e}^{-\left(t+t_{\mathrm{w}}+\tau\right)} \times \\
\left\{\left[\mathrm{I}_{n-1}-\mathrm{I}_{n+1}\right](\tau)\left[\mathrm{I}_{n-1}-\mathrm{I}_{n+1}\right]\left(t+t_{\mathrm{w}}\right)-\left[\mathrm{I}_{n-1}-2 \mathrm{I}_{n}+\mathrm{I}_{n+1}\right](\tau)\left[\mathrm{I}_{n-1}+2 \mathrm{I}_{n}+\mathrm{I}_{n+1}\right]\left(t+t_{\mathrm{w}}\right)\right\}
\end{gathered}
$$

Now, utilizing (A2) and expressing factors of $n$ as derivatives w.r.t. $k$, the Fourier series for $C\left(k ; t, t_{\mathrm{w}}\right)$ may be written in the form:

$$
\begin{gathered}
C\left(k ; t, t_{\mathrm{w}}\right)=\mathrm{e}^{-2\left(t+t_{\mathrm{w}}\right)} \sum_{n} \mathrm{e}^{-i n k}\left[\mathrm{I}_{n}^{2}-\mathrm{I}_{n-1} \mathrm{I}_{n+1}\right]\left(t+t_{\mathrm{w}}\right)-\frac{1}{2} \int_{t-t_{\mathrm{w}}}^{t+t_{\mathrm{w}}} \mathrm{d} \tau \mathrm{e}^{-\left(t+t_{\mathrm{w}}+\tau\right)} \times \\
\left\{\frac{4}{\tau\left(t+t_{\mathrm{w}}\right)} \frac{\partial^{2}}{\partial k^{2}} \sum_{n} \mathrm{e}^{-i n k} \mathrm{I}_{n}(\tau) \mathrm{I}_{n}\left(t+t_{\mathrm{w}}\right)+2\left(\frac{\partial}{\partial \tau}-1\right) \sum_{n} \mathrm{e}^{-i n k} \mathrm{I}_{n}(\tau)\left[\mathrm{I}_{n-1}+2 \mathrm{I}_{n}+\mathrm{I}_{n+1}\right]\left(t+t_{\mathrm{w}}\right)\right\} .
\end{gathered}
$$

All summations in this expression can be evaluated via A5, (A6). Some fairly complicated algebra is required to simplify the resulting expression, but finally one obtains the compact result

$$
\begin{aligned}
C\left(k ; t, t_{\mathrm{w}}\right) & =\mathrm{e}^{-2\left(t+t_{\mathrm{w}}\right)} \frac{\mathrm{I}_{1}\left(2\left(t+t_{\mathrm{w}}\right) \cos \frac{k}{2}\right)}{\left(t+t_{\mathrm{w}}\right) \cos \frac{k}{2}} \\
& +4 \int_{0}^{t_{\mathrm{w}}} \mathrm{d} \tau \mathrm{e}^{-2(t+\tau)}\left[\frac{1}{A} \mathrm{I}_{1}(2 A)+2 \frac{t_{\mathrm{w}}-\tau}{A} \sin ^{2}\left(\frac{k}{2}\right)\left(\mathrm{I}_{1}(2 A)+\frac{t_{\mathrm{w}}-\tau}{A} \mathrm{I}_{2}(2 A)\right)\right],
\end{aligned}
$$

where $A=\sqrt{(t+\tau)^{2} \cos ^{2}(k / 2)+\left(t_{\mathrm{w}}-\tau\right)^{2} \sin ^{2}(k / 2)}$. Eq. (B3) is the most convenient representation for $C\left(k ; t, t_{\mathrm{w}}\right)$, both for numerical and analytical purposes. The calculation of the Fourier transform of the defect susceptibility (21) is comparatively easy; from (A5), (A6) one finds

$$
\chi\left(k ; t, t_{\mathrm{w}}\right)=2 \mathrm{e}^{-2 t}\left\{\left[\mathrm{I}_{0}+\mathrm{I}_{1}\right](2 t)-\mathrm{I}_{0}(2 A)-\frac{t \cos ^{2}(k / 2)+t_{\mathrm{w}} \sin ^{2}(k / 2)}{A} \mathrm{I}_{1}(2 A)\right\},
$$

with $A=\sqrt{t^{2} \cos ^{2}(k / 2)+t_{\mathrm{w}}^{2} \sin ^{2}(k / 2)}$. 


\section{APPENDIX C: POWER-LAW COVARIANCES}

Here we show that the covariances $q_{\mathrm{P}, n}$ given in (25) follow a power-law as $|n| \rightarrow \infty$ and establish the link $q_{\mathrm{P}, n}=\mathcal{F}^{-1}\left\{q_{\mathrm{P}}(k)\right\}$. Let us first focus on the Fourier integral (22) which - since $q_{\mathrm{P}}(k)$ is even in $k$ - may be written as

$$
q_{\mathrm{P}, n}=\frac{\Gamma^{2}\left(\frac{1+\alpha}{2}\right)}{2^{1-\alpha} \Gamma(\alpha)} \int_{0}^{2 \pi} \frac{\mathrm{d} k}{2 \pi}\left(\sin \frac{k}{2}\right)^{\alpha-1} \cos (n k)
$$

where $0<\alpha<1$ as before. The simple substitution $x=k / 2$ yields the solvable integral [40]

$$
\int_{0}^{\pi} \frac{\mathrm{d} x}{\pi}(\sin x)^{\alpha-1} \cos 2 n x=\frac{(-1)^{n}}{\alpha 2^{\alpha-1} \mathrm{~B}\left(\frac{1+\alpha}{2}+n, \frac{1+\alpha}{2}-n\right)} .
$$

Using the functional relation [40] $\mathrm{B}(x, y)=\Gamma(x) \Gamma(y) / \Gamma(x+y)$ for the Beta function $\mathrm{B}(x, y)$ and simplifying the remaining expression yields the result for $q_{\mathrm{P}, n}$ given in (25). Now we turn to the asymptotic behaviour of $q_{\mathrm{P}, n}$ as $|n| \rightarrow \infty$. For $n \geq 1$ we may rewrite $q_{\mathrm{P}, n}$, using $\Gamma(x) \Gamma(1-x)=$ $\pi / \sin \pi x$ and $\Gamma(x+1)=x \Gamma(x)$, in the form

$$
q_{\mathrm{P}, n}=\prod_{k=0}^{n-1} \frac{1-\alpha+2 k}{1+\alpha+2 k}
$$

It is obvious from (C3) that $q_{\mathrm{P}, n}$ is monotonically decreasing and vanishes for $n \rightarrow \infty$ as long as $\alpha>0$. It is equally clear that $q_{\mathrm{P}, n}=1$ for $\alpha \rightarrow 0$ and $q_{\mathrm{P}, n}=\delta_{n, 0}$ as $\alpha \rightarrow 1$ (since $q_{\mathrm{P}, n}$ is even in $n$ and $\left.q_{\mathrm{P}, 0}=1 \forall \alpha\right)$. In order to understand the asymptotic behaviour of $q_{\mathrm{P}, n}$ we take the logarithm of (C3) and use the bounds

$$
\int_{0}^{n} \mathrm{~d} k a_{k} \leq \sum_{k=0}^{n-1} a_{k} \leq a_{0}+\int_{0}^{n-1} \mathrm{~d} k a_{k}
$$

which hold for any non-increasing function $a_{k}$. For the case at hand the integrals can be solved easily. Exponentiating the result, multiplying by $n^{\alpha}$ and taking the limit $n \rightarrow \infty$ then gives

$$
(2 \mathrm{e})^{-\alpha} \sqrt{\frac{(1-\alpha)^{1-\alpha}}{(1+\alpha)^{1+\alpha}}} \leq \lim _{n \rightarrow \infty} n^{\alpha} q_{\mathrm{P}, n} \leq(2 \mathrm{e})^{-\alpha} \sqrt{\frac{(1+\alpha)^{1-\alpha}}{(1-\alpha)^{1+\alpha}}}
$$

which implies that there exists a finite constant $c$ such that $q_{\mathrm{P}, n} \sim c n^{-\alpha}$ for $0<\alpha<1$.

[1] L. F. Cugliandolo and J. Kurchan, Phys. Rev. Lett. 71, 173 (1993).

[2] J.-P. Bouchaud, L. F. Cugliandolo, J. Kurchan and M. Mézard, in Spin glasses and random fields, Ed.: A. P. Young (World Scientific, Singapore, 1998).

[3] L. F. Cugliandolo, Lecture Notes, Les Houches, (July 2002), preprint cond-mat/0210312

[4] L. F. Cugliandolo and J. Kurchan, J. Phys. A: Math. Gen. 27, 5749 (1994).

[5] S. Franz and M. Mézard, Europhys. Lett. 26, 209 (1994); Physica A 210, 48 (1994).

[6] L. F. Cugliandolo, J. Kurchan and L. Peliti, Phys. Rev. E 55, 3898 (1997).

[7] A. Barrat, Phys. Rev. E 57, 3629 (1998).

[8] L. Berthier, J.-L. Barrat and J. Kurchan, Eur. Phys. J. B 11, 635 (1999).

[9] P. Sollich, F. Lequeux, P. Hébraud, and M. E. Cates, Phys. Rev. Lett. 78, 2020 (1997).

[10] L. Berthier, J.-L. Barrat and J. Kurchan, Phys. Rev. E 61, 5464 (2000).

[11] A. J. Bray, Adv. Phys. 43, 357 (1994).

[12] Th. M. Nieuwenhuizen, Phys. Rev. Lett. 80, 5580 (1998).

[13] S. Fielding and P. Sollich, Phys. Rev. Lett. 88, 050603 (2002).

[14] L. Berthier and J.-L. Barrat, Phys. Rev. Lett. 89, 095702 (2002); J. Chem. Phys. 116, 6228 (2002).

[15] L. F. Cugliandolo, J. Kurchan and G. Parisi, J. Phys. I (France) 4, 1641 (1994).

[16] L. F. Cugliandolo and D. S. Dean, J. Phys. A: Math. Gen. 28, 4213 (1995). 
[17] W. Zippold, R. Kühn, and H. Horner, Eur. Phys. J. B 13, 531 (2000).

[18] S. A. Cannas, D. A. Stariolo, and F. A. Tamarit, Physica A 294, 362 (2001).

[19] F. Corberi, E. Lippiello, and M. Zannetti, Phys. Rev. E 65, 046136 (2002).

[20] G. Parisi, F. Ricci-Tersenghi and J. J. Ruiz-Lorenzo, Eur. Phys. J. B 11, 317 (1999).

[21] D. A. Stariolo and S. A. Cannas, Phys. Rev. B 60, 3013 (1999).

[22] F. Corberi, E. Lippiello, and M. Zannetti, Phys. Rev. E 63, 061506 (2001); F. Corberi, E. Lippiello and M. Zannetti, Eur. Phys. J. B 24, 359 (2001); F. Corberi, C. Castellano, E. Lippiello, and M. Zannetti, Phys. Rev. E 65, 066114 (2002).

[23] M. Henkel, M. Pleimling, C. Godrèche, and J.-M. Luck, Phys. Rev. Lett. 87, 265701 (2001).

[24] M. Henkel and M. Pleimling (submitted); F. Corberi, E. Lippiello, and M. Zannetti, preprint cond-mat/0211609

[25] M. Henkel, M. Paessens, and Michel Pleimling, preprint cond-mat/0211583

[26] C. Godrèche and J.-M. Luck, J. Phys. A: Math. Gen. 33, 1151 (2000).

[27] E. Lippiello and M. Zannetti, Phys. Rev. E 61, 3369 (2000).

[28] G. J. M. Koper and H. J. Hilhorst, Physica A 155, 431 (1989).

[29] C. Godrèche and J.-M. Luck, J. Phys. A: Math. Gen. 33, 9141 (2000).

[30] C. Godrèche and J.-M. Luck, J. Phys. C 14, 1589 (2002).

[31] P. C. Hohenberg and B. I. Halperin, Rev. Mod. Phys. 49, 435 (1977).

[32] L. Berthier, P. C. W. Holdsworth and M. Sellitto, J. Phys. A: Math. Gen. 34, 1805 (2001).

[33] P. Calabrese and A. Gambassi, Phys. Rev. E 66, 066101 (2002).

[34] P. Calabrese and A. Gambassi, preprint cond-mat/0207487

[35] P. Calabrese and A. Gambassi, preprint cond-mat/0211062

[36] A. Buhot and J.P. Garrahan, Phys. Rev. Lett. 88, 225702 (2002).

[37] A. J. Bray, J. Phys. A 23, L67 (1989).

[38] P. Mayer and P. Sollich, in preparation.

[39] P. Sollich, S. Fielding, and P. Mayer, J. Phys. C 14, 1683 (2002).

[40] I. Gradstein and I. Ryzhik, Table of Integrals, Series, and Products, Academic Press (1980).

[41] For $t=t_{\mathrm{w}}$ the integral in (18) is of the form of a Laplace convolution. A Laplace transform indeed allows one to simplify this expression and rewrite it explicitly in terms of modified Bessel functions.

[42] See e.g. B. Derrida, R. Zeitak, Phys. Rev. E 54, 2513 (1996).

[43] J. E. Santos, J. Phys. A 30, 3249 (1997).

[44] K. P. N. Murthy and G. M. Schütz, Phys. Rev. E 57, 1388 (1998).

[45] P. Mayer, Ph.D. thesis, King's College London (unpublished).

[46] J. L. Spouge, Phys. Rev. Lett. 60, 871 (1998); J. G. Amar and F. Family, Phys. Rev. A 41, 3258 (1990).

[47] G.H. Fredrickson and H.C. Andersen, Phys. Rev. Lett. 53, 1244 (1984). 